\title{
1 Genomically hardwired regulation of gene activity orchestrates cellular \\ 2 iron homeostasis in Arabidopsis
}

4 En-Jung Hsieh ${ }^{1}$, Wen-Dar Lin ${ }^{1}$, and Wolfgang Schmidt ${ }^{1,2,3, *}$

$6 \quad{ }^{1}$ Institute of Plant and Microbial Biology, Academia Sinica, Taipei 11529, Taiwan

$7 \quad{ }^{2}$ Biotechnology Center, National Chung-Hsing University, Taichung 40227, Taiwan

$8{ }^{3}$ Genome and Systems Biology Degree Program, College of Life Science, National Taiwan

9 University, Taipei 10617, Taiwan

10 *corresponding author. E-mail: wosh@gate.sinica.edu.tw

\section{Abstract}

13 Iron $(\mathrm{Fe})$ is an essential micronutrient that plays pivotal roles as electron donor and catalyst

14 across organisms. In plants, variable, often insufficient Fe supply necessitates mechanisms

15 that constantly attune Fe uptake rates and recalibrate cellular Fe homeostasis. Here, we show

16 that short-term $(0.5,6$, and $12 \mathrm{~h})$ exposure of Arabidopsis thaliana plants to Fe deficiency

17 triggered massive changes in gene activity governed by transcription and alternative splicing

18 (AS), regulatory layers that were to a large extent mutually exclusive. Such preclusion was

19 not observed for genes that are directly involved in the acquisition of Fe, which appears to be

20 concordantly regulated by both expression and AS. Generally, genes with lower splice site

21 strengths and higher intron numbers were more likely to be regulated by AS, no dependence

22 was on gene architecture was observed for transcriptionally controlled genes. Conspicuously,

23 specific processes were associated with particular genomic features and biased towards either

24 regulatory mode, suggesting that genomic hardwiring is functionally biased. Early changes in

25 splicing patterns were, in many cases, congruent with later changes in transcript or protein 
26 abundance, thus contributing to the pronounced transcriptome-proteome discordance

27 observed in plants.

\section{Introduction}

30 Iron $(\mathrm{Fe})$ is mineral nutrient with a plethora of vital functions across all kingdoms of life. In

31 plants, $\mathrm{Fe}$ is a critical component of electron chains in photosynthesis and required for the

32 biosynthesis of chlorophyll. Iron is abundant in the Earth's crust, but its availability is often

33 limited by interaction with other soil constituents, in particular at high redox and $\mathrm{pH}$ values.

34 In most aerated soils, Fe is present in the form of sparingly soluble $\mathrm{Fe}$ (III) oxides which

35 cannot readily be taken up by plants, causing leaf chlorosis, reduced yield, and decreased

36 quality of edible plant parts. Low plant Fe content can jeopardise human health by causing

37 Fe-deficiency anaemia, in particular in populations with a predominantly plant-based diet. To

38 counteract limited Fe availability, plants have evolved a suite of mostly transcriptionally

39 regulated responses that mediate the acquisition of Fe from recalcitrant pools in soils ${ }^{1,2}$. In

40 contrast to graminaceous species, which take up $\mathrm{Fe}^{3+}$ after secretion of $\mathrm{Fe}$-avid

41 phytosiderophores (strategy II) ${ }^{3}$, dicotyledonous plants acquire $\mathrm{Fe}^{2+}$ by the concerted action

42 of processes that mobilize sparingly soluble $\mathrm{Fe}^{3+}$ by protonation, chelation, and reduction in

43 response to imbalances caused by inadequate $\mathrm{Fe}$ supply (strategy $\mathrm{I})^{4-8}$. The responses to $\mathrm{Fe}$

44 starvation are controlled by a complex network of homeostatic mechanisms that orchestrate

45 the acquisition, transport, and cellular homeostasis of $\mathrm{Fe}^{9-12}$.

46 Due to the central role of ferrous Fe as an electron donor and catalyst, its absence or

47 insufficiency compromises energy production and causes severe metabolic perturbations.

48 Reduced respiration efficiency and impaired activity of Fe-containing key enzymes of the

49 tricarboxylic acid (TCA) cycle such as aconitase or succinate dehydrogenase constitute major

50 constraints in Fe-undernourished cells, necessitating extensive rerouting of carbon flow 
51 during periods of low Fe availability. Increased glycolysis rates upon Fe starvation, for

52 instance, have been described for diverse eukaryote systems such as human cell lines ${ }^{13}$,

53 macrophages ${ }^{14}$, and yeast ${ }^{15}$, suggesting that enhanced glycolytic flux is a conserved

54 mechanism to compensate for decreased respiration. In Fe-deficient plants, pronounced

55 metabolic changes have been observed across species ${ }^{16-23}$, which, with some notable

56 exceptions, are not mirrored in transcriptomic profiles. The mechanisms that govern such

57 metabolic rerouting have remained largely elusive.

58 Alternative splicing (AS) of pre-mRNAs, i.e., the process of selecting different

59 combinations of splice sites for intron removal and exon ligation, contributes to the diversity

60 of transcripts and proteins by producing multiple mRNA and protein isoforms, and may, at

61 least partly, be causative for the lack of transcriptional footprints of Fe deficiency-induced

62 alterations in central carbon metabolism. In contrast to animals, where exon skipping (ES) is

63 dominating over other forms of AS, in plants, intron retention (IR) and alternative donor or

64 acceptor splice sites (DA) are the prevalent forms of $\mathrm{AS}^{24-26}$. While ES is likely to produce

65 functional proteins, the latter forms of AS often interrupt the open reading frame and lead to

66 the introduction of premature stop codons, causing the formation of aberrant proteins or

67 mRNA that is targeted to the nonsense-mediated decay pathway ${ }^{27}$. Between $60-80 \%$ of the

68 multi-exonic plant genes are alternatively spliced, a percentage that can vary in response to

69 environmental stimuli or stress ${ }^{28-30}$. Thus, in plants, AS constitutes a huge and mostly

70 unexplored source of gene regulation of undisclosed significance, possibly fine-tuning the

71 transcriptome to the prevailing environmental conditions.

How environmental information is communicated into the nucleus to alter pre-mRNA

73 splicing patterns remains largely enigmatic. AS is driven by a large suite of mRNA-binding,

74 spliceosome-associated proteins such as serine/arginine-rich (SR) proteins and heterogeneous

75 nuclear ribonucleoproteins (hnRNPs) that binds to cis-regulatory sequences on the pre- 
76 mRNA. Splicing factors are altered in abundance, localization, and activity upon stress,

77 conferring plasticity to the patterns of alternative splicing in response to internal or external

78 stimuli $^{31-35}$. Moreover, chromatin-related factors such as histone modifications and

79 nucleosome positioning, and genomic traits such as gene body length and splice site strength

80 were shown to affect $\mathrm{AS} 30^{30,36-38}$.

81 By employing short-term exposure of Arabidopsis plants to Fe deficiency as a well-

82 explored environmental cue, we report here that the type of gene regulation (i.e., AS or

83 differential expression) is determined by the architecture of the gene, the amplitude of the

84 response, and the temporal pattern of the changes in gene activity. Genomic features are

85 biased towards and typical of specific Fe-responsive processes and seem to govern AS but

86 not transcriptional regulation, which appears to be chiefly driven by physiological

87 requirements. While differential AS (DAS) and differential gene expression (DE) are

88 generally complementary forms of gene regulation, we found this rule suspended when core

89 genes of the Fe deficiency response are considered, suggesting that the two regulatory modes

90 can operate synergistically to allow for an optimally tuned response. We further found that, in

91 many but not all cases, DAS features observed early after the onset of Fe-deficient conditions

92 represent a blueprint of what becomes evident at the activity, transcript, or protein level at

93 later stages of Fe deficiency.

95 Results

96 Iron deficiency triggers rapid changes in transcription and splicing patterns

97 To gain insights into transcriptional and post-transcriptional alterations in response to $\mathrm{Fe}$

98 deficiency, Arabidopsis plants were subjected to $0.5,6$, and 12 hours of growth in Fe-free

99 media and subsequent transcriptomic profiling against plants that were transferred to fresh

100 Fe-replete nutrient solution using the RNA-seq methodology. The Illumina HiSeq 4000 
101 sequencing system was adopted to acquire approximately 70-80 million reads for each library

102 with a read length of 100 base pairs (Supplementary Table S1). On average, a total of circa

10327,000 genes was expressed in both roots and shoots, of which subsets of 1,161 (roots) and

1041,027 (shoots) were defined as differentially expressed between Fe-deficient and Fe-

105 sufficient control plants at one or more of the three sampling time points with relevant

106 expression levels (RPKM > the square root of the mean expression value of the whole

107 dataset), $P<0.05$, and a fold-change $>2$ after normalization using the TMM (Trimmed Mean

108 of M-values) method (Fig. 1a). In both roots and shoots, differentially expressed genes

109 (DEGs) showed a relatively small overlap among the experimental time points, indicative of

110 a highly dynamic and temporally distinctive response to Fe deficiency (Fig. 1c).

111 Changes in the pattern of alternative splicing (AS) upon Fe deficiency were analysed

112 with the aid of the Read Analysis and Comparison Kit in Java (RACK J) software toolbox ${ }^{39}$.

113 Applying the same thresholds used for the classification of DE genes and considering only

114 genes in which the AS feature was observed in all three replicates, a subset of 7,517 genes

115 was classified as differentially alternatively spliced (DAS), exceeding the number of

116 differentially expressed genes (DEGs) by more than fourfold (Fig. 1f). The largest fraction of

117 DAS genes (82\%) produced transcripts that harboured differential intron retention (DIR)

118 features, a smaller subset (15\%) carried differential donor/acceptor (DDA) sites, and 2.8\% of

119 the DAS transcripts exhibited differential exon skipping (DES) after exposure to Fe

120 deficiency (Fig. 1e). A considerable subset of DAS genes carried two or more different AS

121 features. Genes with DAS features showed distinct overlaps among the different

122 experimental timepoints which depended on the type of AS with DIR being more conserved

123 than DEG and the overlap of DDA genes comparable to that observed for DEGs (Fig. 1c, d).

124 With the exception of DES, in which enhanced and repressed features varied over time, DAS

125 showed a neutral outcome of splicing efficiency upon exposure to Fe deficiency, with about 
126 similar proportions of genes carrying enhanced or reduced splicing features (Supplementary

127 Fig. S1).

\section{Fe deficiency induces a series of concatenated responses in roots and shoots}

130 Transfer of plants to Fe-deplete media triggered the consecutive induction of a suite of

131 distinctly timed responses (Fig. 2). Induction of these processes was almost simultaneously

132 observed in roots and shoots, suggesting that minor changes in Fe supply suffice to sense Fe

133 deficiency in all plant parts. After six hours of exposure to Fe-deficient conditions, induction

134 of the basic Fe uptake machinery comprising the Fe chelate reductase FRO2 and the $\mathrm{Fe}^{2+}$

135 transporter IRT1, vacuolar sequestration of excessive cytosolic levels of secondary-substrate

136 metal cations such as $\mathrm{Mn}^{2+}$ and $\mathrm{Zn}^{2+}$, and downregulation of $\mathrm{Fe}$ import into the vacuole were

137 the most prominent transcriptionally regulated responses in roots (Fig. 2a). Induction of these

138 processes was accompanied by increased expression of a suite of genes encoding regulators

139 such as the IRONMAN peptides IMAI and IMA2 and the transcription factor POPEYE (PYE)

140 (Fig. 2a). A module that was induced slightly later comprised genes mediating the

141 mobilization of $\mathrm{Fe}$ in the rhizosphere via secretion of protons and Fe-mobilizing coumarins

142 (Fig. 2b). Induction of Fe mobilisation was accompanied by increased expression of the

143 transcription factors $M Y B 72$ and $M Y B 10$, which were shown to be crucial for plant survival in

144 soils with severely restricted Fe availability (Fig. 2 b) ${ }^{40}$. Notably, sampling at very early

145 stages of Fe deficiency $(0.5 \mathrm{~h})$ revealed expression changes in a direction which was

146 antagonistic to that observed at later stages, suggesting that rearrangements of the

147 transcriptional machinery cause transient perturbations in gene expression that later result in

148 robust transcriptional regulation of these genes (Supplementary Tables S2-5).

149 In shoots, exposure to Fe-deplete media for 6 hours induced Fe transport across the

150 plasma membrane via OPT3 and IRT3 and expression of a variety of regulators such as PYE, 
151 the E3 ligase BRUTUS (BTS), subgroup Ib bHLH proteins, and IMA1-IMA3 (Fig. 2c). Similar

152 to root cells, sequestration of transition metals into the vacuole was induced at this time point.

153 In addition, altered transcription of genes involved in ROS homeostasis (CGLD27, ENH1)

154 was observed in shoots (Supplementary Table S2). After 12 hours, increased trans-plasma

155 membrane transport of Fe-nicotianamine (NA) by YSL1 and $\mathrm{Cu}^{2+}$ via COPT2 can be inferred

156 from the induction of the cognate genes at this time-point. Also, chlorophyll metabolism

157 (HEMA1, NYCl) and ROS homeostasis (NEET) were affected by growth on Fe-deplete

158 media (Fig. 2d; Supplementary Table S2).

160 In addition to genes directly involved in Fe homeostasis, in roots, and to a lesser extent in

161 shoots, short-term exposure to Fe deficiency caused a dramatic increase in the transcription

162 of genes associated with jasmonic acid (JA) biosynthesis and signalling (Fig. 3;

163 Supplementary Table S2 and S3), a response which was strictly restricted to the 6-hour time

164 point. In particular, the first steps of JA biosynthesis and the expression of a suite of

165 transcriptional regulators, referred to as jasmonate ZIM-domain (JAZ) proteins, were

166 strongly induced in response to Fe deficiency (Fig. 3).

\section{DAS orchestrates rerouting of the central carbon metabolism}

169 Glycolysis is a key process in energy production and provides metabolic intermediates for

170 biosynthetic processes, storage, and anaplerotic processes. A subset of 75 genes associated

171 with glycolysis was regulated by the Fe regime, almost exclusively by DAS (Supplementary

172 Table S4). Notable exceptions from this pattern were the first and the last step in glycolysis,

173 catalysed by phosphofructokinase (PFK) and pyruvate kinase (PK), which are robustly

174 upregulated at later stages of iron deficiency in transcriptional surveys ${ }^{9,11}$. Analysis of PFK1

175 expression by RT-qPCR showed increasing induction over the first three days of Fe 
176 deficiency in roots and, to a much lesser extent, in shoots (Fig. 4a, c). Expression of the Fe

177 status marker $b H L H 38$ was increased over the first three days of Fe deficiency with a higher

178 transcript level in shoots, indicating that the lower induction of PFK1 in shoots was not

179 associated with a healthier Fe status of leaf cells (Fig. 4c). Since PFK is catalysing the rate-

180 limiting step in glycolysis, it can be assumed that glycolytic flux is particularly increased in

181 root cells. The final step in glycolysis, the PK-mediated conversion of phosphoenolpyruvate

182 (PEP) to pyruvate, was repressed in roots via enhanced IR of the cytosolic PK isoform

183 At5g08570. In shoots, transcripts of this isoform carried reduced IR upon exposure to Fe

184 deficiency. RT-qPCR analysis revealed decreased transcript abundance of this isoform in

185 both roots and shoots one day after exposure to Fe-deplete media (Fig. 4d). Downregulation

186 of another PK-encoding gene in roots (At3g49160) was reported in several transcriptomic

187 studies $^{7,11}$, further supporting the supposition that PK activity is decreased upon Fe deficiency.

188 For several steps of root glycolysis, DAS appear to precede changes in enzyme

189 activity or abundance. Fructose-bisphosphate aldolase (FBA) catalyses the reversible aldol

190 cleavage of fructose-1,6-bisphosphate, yielding dihydroxyacetone phosphate. In roots, the

191 cytosolic isoform FBA8 exhibited both repressed IR and DA features (Fig. 4a). Also, the

192 cytosolic phosphoglycerate mutase (PGM) isoforms $i P G A M 1$ and $i P G A M 2$ showed reduced

193 IR upon Fe deficiency (Fig. 4a). In a previous study, we observed increased phosphorylation

194 of FBA8 and iPGAM1 protein and accumulation of the (cytosolic) FBA4 protein in roots

195 upon prolonged Fe deficiency ${ }^{23,41}$, indicating Fe deficiency-induced increase in enzyme

196 activity at later stages of Fe deficiency. Moreover, FBA and PGM accumulated in Fe-

197 deficient roots of Beta vulgaris and Cucumis sativa ${ }^{19,42}$, suggesting that increased abundance

198 of these enzymes in response to Fe deficiency is conserved across strategy-I species.

199 The anti-directional regulation of PFK and PK in roots of Fe-deficient plants implies

200 imbalances of glycolysis intermediates under conditions of Fe starvation. To further 
201 investigate this matter, we determined the concentrations of glucose-6-phosphate (G6P),

202 phosphoenolpyruvate (PEP), and pyruvate over the first three days of Fe deficiency via

203 UHPLC-MS analysis. In roots, but not in shoots, the level of both PEP and pyruvate was

204 increased three days after the onset of Fe deficiency, indicating severe perturbances of

205 pyruvate metabolism at later stages of Fe deficiency (Fig. 4b). Other glycolysis intermediates

206 such as G6P or fructose-6-phosphate (F6P) did neither show pronounced changes in roots nor

207 in shoots upon short-term or extended exposure to Fe deficiency (Fig. 4b), suggesting that the

208 concentrations of theses intermediates are in steady-state during Fe deficiency.

\section{The TCA cycle malfunctions in roots of Fe-deficient plants}

211 A subset of 34 of TCA-related genes was responsive to the Fe regime (Supplementary Table

212 S5). Similar to what has been observed for glycolysis, genes encoding enzymes of the TCA

213 cycle showed limited transcriptional control and were predominantly regulated by DAS

214 (Supplementary Table S5). In roots, all TCA cycle metabolites under study accumulated

215 three days after the onset of Fe deficiency, with citrate, malate, and succinate being most

216 abundant (Supplementary Fig. S3). In particular, citrate levels were strongly increased; no

217 such accumulation was observed in shoots. Citrate is synthesized in the first committed and

218 pace-making step of the cycle by condensation of oxaloacetate and acetyl CoA, catalysed by

219 citrate synthase (CSY). However, the mitochondrial isoforms CSY4 and CSY5 were neither

220 regulated by DAS nor by DE, suggesting other causes for the increased citrate levels.

221 Aconitase (ACO), catalysing the subsequent conversion of citrate to isocitrate, harbours an

222 active $\left[\mathrm{Fe}_{4} \mathrm{~S}_{4}\right]^{2+}$ cluster, which may compromise ACO activity in Fe-deficient plants and

223 contribute to the accumulation of citrate. In line with this assumption, ACO2 and ACO3 were

224 transcriptionally downregulated in roots three days after transfer to Fe-deficient media ${ }^{7,9,43}$,

225 indicating restricted conversion of citrate into isocitrate. Also other enzymes of the TCA 
226 cycle such as citrate synthase and isocitric dehydrogenase were shown to affect by the iron

227 regime ${ }^{13}$, further supporting the notion that the TCA cycle is compromised or truncated under

228 conditions of Fe deficiency. This scenario is supported by a lack of consistent and mostly

229 repressive DAS-regulation of all steps of the TCA cycle (Supplementary Fig. S3).

231 The routes of pyruvate metabolization differ between roots and shoots

232 Pyruvate derived from glycolysis can be either converted to acetyl-CoA by pyruvate

233 dehydrogenase (PDH) in the mitochondrial matrix, or decarboxylated to acetaldehyde

234 through pyruvate decarboxylase (PDC) in the cytosol. PDH is a multienzyme complex

235 composed of three enzymes, pyruvate dehydrogenase (E1), dihydrolipoyl transacetylase (E2),

236 and dihydrolipoyl dehydrogenase (E3). In roots, isoforms of all three enzymes showed

237 generally reduced IR upon Fe deficiency (Fig. 5a). In shoots, a more complex, mostly

238 repressive regulation was observed (Fig. 5b). Alcohol dehydrogenase (ADH) reduces

239 pyruvate-derived acetaldehyde to ethanol and $\mathrm{NAD}^{+}$. In roots, short-term exposure to $\mathrm{Fe}$

240 deficiency resulted in repressed IR of both $P D C 1$ and $A D H 1$ transcripts, indicative of

241 increased ethanolic fermentation (Fig. 5a). Also, the class III type alcohol dehydrogenase

242 ADH2 (HOT5) and the putative ADH At4g22110 showed DAS features that were mostly

243 repressed upon Fe starvation. By contrast, in shoots $A D H I$ carried chiefly enhanced DIR

244 features (Fig. 5b). Determination of $A D H 1$ transcript levels in roots by RT-qPCR revealed a

245 steep increase after two days of Fe deficiency (Supplementary Fig. S4), matching

246 transcriptomic studies carried out at later stages on roots of Fe-deficient plants ${ }^{7,44}$. These data

247 suggest that the ADH-mediated fermentation route is supported in roots, but not in shoots of

248 Fe-deficient plants.

249 An alternative fate of acetealdehyde is the conversion to acetic acid, catalysed by a

250 group of aldehydedehydrogenases (ALDHs) in a reaction yielding NADH. In shoots, three 
251 NAD-dependent ALDHs (the mitochondrial family 2 proteins ALDH2B7 and ALDH2B4,

252 and the cytoplasmatic family 3 protein ALDH3H1) showed reduced IR upon Fe starvation

253 (Fig. 5b). In roots, $A L D H 2 B 7$ transcripts carried enhanced IR at two time points after the

254 onset of Fe-deficient conditions (Fig. 5a), indicating repressed acetic acid formation. It thus

255 appears that different fermentation routes are engaged in roots and shoots, possibly driven by

256 differences in redox regulation and energy status of leaf and root cells.

257 Besides reduced PK activity, the build-up of toxic pyruvate concentrations in roots of

258 Fe-deficient plants is circumvented by rapid metabolization of its precursor, PEP. PEP is a

259 potent inhibitor of PFK activity and, if present at high levels, decreases glycolysis rates ${ }^{45-47}$.

260 Increased $\beta$-carboxylation of PEP via PEP carboxylase (PPC) in roots is a hallmark of Fe-

261 deficient roots of strategy I plants ${ }^{17}$. In roots, PPC1 transcripts carried reduced IR features

262 (Fig. 5a), transcription of the gene was induced at later stages of Fe deficiency ${ }^{7}$. The reverse

263 reaction, the conversion of oxaloacetate to PEP by PEP carboxykinase (PCK), showed

264 enhanced IR (Fig. 5a,b). In concert with these results, $P C K 1$ was found to be downregulated

265 in roots (but not in shoots) of plants subjected to Fe deficiency for three days ${ }^{7,48}$.

\section{DE and DAS concertedly regulate the ferrome}

268 Strikingly, the vast majority of DAS-regulated genes did not change significantly in

269 expression over the experimental period. In total, less than $1 \%$ of the DAS genes were also

270 differentially expressed (Fig. S1e). Plotting fold-changes of the different DAS types versus

271 DE revealed a moderate correlation between DIR and DE (Fig. 6a). By contrast, regulation of

272 gene activity by DDA and DE was almost mutually exclusive (Fig. 6b).

273 The principle of mutual exclusivity does not seem to apply when only genes that were

274 shown to robustly respond to Fe deficiency at the transcriptional level in previous studies are

275 considered, a subset that has been referred to as the 'ferrome' ${ }^{49}$. To revise and update this 
276 definition, we surveyed recent public transcriptomic datasets of Fe-deficient plants derived

277 from RNA-seq profiling studies. For roots, seven datasets were analysed, and genes that were

278 found to be differentially expressed in at least four of these studies (three out of five in shoots)

279 were included in the Arabidopsis ferrome. This procedure yielded subsets of 108 and 100

280 genes in roots and shoots, respectively; a suite of 39 genes was robustly differentially

281 expressed in both roots and shoots (Supplementary Table S6). From 114 DE-regulated

282 ferrome genes, a subsection of $42(37 \%)$ was additionally regulated by DAS (Supplementary

283 Table S2), a percentage that is substantially higher than the average of 3-5\% observed in

284 roots at the different sampling points. Most of the genes involved in Fe uptake were

285 transcriptionally regulated; however, some regulators (e.g., GRF11, BSTL2), genes encoding

286 enzymes such as the $\beta$-glucosidase $B G L U 42$ which is critical for the deglycosylation of

287 coumarins prior to secretion ${ }^{50}$, and the $\mathrm{Zn} / \mathrm{Fe}$ transporter IRT3, were exclusively regulated by

288 DAS. All of these genes were shown to be transcriptionally induced at later stages of Fe

289 deficiency (Supplementary Table S2).

290 Unexpectedly, in both roots and shoots the percentage of DE-regulated ferrome genes

291 increased over time. While after 0.5 hours the majority of Fe-responsive genes was DAS-

292 regulated, the proportions of DE and DAS were about equal at 6 hours and massively shifted

293 towards transcriptional regulation at 12 hours (Fig. 7a, b). A comparison with a previous

294 survey of genes responsive to a 3-day-period of Fe starvation revealed a steep decrease in the

295 percentage of DAS-regulated genes at later stages of Fe deficiency (Fig. 7b). Comparing

296 different subgroups within the ferrome showed that transcripts encoding enzymes tended to

297 have a higher percentage of DAS than transporters and regulators. After 3 days,

298 transcriptional regulation of metabolic genes was still lower than in the other categories, but

299 the participation of DAS in the overall gene regulation was dramatically reduced to about

300 relative to what was observed in the short term (Fig. 7b). Such decreased participation of 
301 DAS in the regulation of Fe homeostasis was also observed when other gene ontologies were

302 considered (Fig. 7c). This analysis also revealed that at later stages of Fe deficiency

303 transcriptional regulation of ferrome genes is prioritized over other processes, indicating that

304 a more severely disturbed Fe metabolism prioritises expression of a specific subset of genes

305 to secure efficient Fe acquisition. It may also be assumed that at this stage, early DAS events

306 have been established as stable changes in protein abundance, which is not monitored in the

307 two transcriptomic studies considered here.

309 Gene architecture predefines the type of gene regulation

310 From the survey of Fe-responsive genes, it appears that the various Fe-responsive processes

311 are preferentially controlled by either DE (e.g., JA-biosynthesis and signalling), DAS

312 (glycolysis and TCA cycle), or both DE and DAS (Fe uptake), regulatory patterns that are

313 possibly associated with the amplitude of the response (Supplementary Tables S2-5). To

314 investigate whether genomic traits influence or govern the type of gene regulation, we first

315 analysed the number and length of introns of Fe-responsive genes. Intron length was only

316 moderately correlated with the probability of IR. Introns with a length of more than $200 \mathrm{bp}$

317 had a slightly higher chance of being retained upon Fe deficiency than shorter introns,

318 reaching a peak at about 700 bp (Supplementary Fig. S2a). The number of introns, on the

319 other hand, appeared to be a strong predictor for AS. When normalized to the average intron

320 number across the genome, the number of genes producing transcripts with DIR, DDA, or

321 DES features showed a steep incline which saturated at an intron number between 10 and 20

322 (Supplementary Fig. S2b, c). Notably, intron-rich genes harbouring >50 introns were rarely

323 found to be subject to DAS (Supplementary Fig. S2b, c).

324 As further parameters that presumptively affect the type of gene regulation, we

325 investigated the influence of 5' and 3' splice site strength, promoter length, and the number 
326 of transcription factor binding sites (TFBSs) on DE and DAS. Plotting the minimum average

327 splice site strength [ASS; $\left.\left(\min 5^{\prime}+\min 3^{\prime}\right) / 2\right]$ of Fe-responsive genes against the intron

328 number showed that weak splice sites were generally associated with lower intron numbers

329 (Fig. 8a-c), a finding that may be causal rather than merely correlative. Considering DE- and

330 DAS-regulated genes separately revealed that the average intron number was much lower in

331 DE than in DAS genes, while ASS appears to be biased towards higher values (i.e., stronger

332 splice sites) in this group relative to DAS-regulated genes. With few exceptions, genes with

333 intron numbers $>20$ were regulated by DAS (Fig. 8b). However, highly negative ASS values

334 do not appear to compromise transcriptional regulation, indicating that DE can be

335 promiscuously employed to regulate genes hardwired for being DAS-regulated. Factors that

336 may support transcriptional regulation such as promoter length and the number of

337 transcription factor binding sites (TFBSs) did not pose major effects on gene expression.

338 While promotor length was correlated with the number of TFBSs, no bias towards higher

339 values was observed for DE-regulated genes, suggesting that these parameters are not

340 decisive for the type of regulation (Fig. 8c, d). It thus appears that DE is not dictated by

341 genomic features and can be called into play when more robust regulation is required.

343 The type of gene regulation is genomically hardwired across biological process

344 To further investigate the mechanisms underlying the regulation of Fe-responsive genes, we

345 compared the genetic architecture of genes involved in various processes that are affected by

346 the Fe regime. Homing in on individual genes of the ferrome, a comparison of the DE- and

347 DAS-regulated subsets supported the trend observed for all Fe-responsive genes, i.e., a

348 marked shift towards stronger splice sites for DE-regulated genes (Fig. 9). However,

349 regulation by DE was also observed for genes with very weak (i.e., highly negative) splice

350 sites and high intron numbers (Fig. 9a). The difference in ASS between DE- and DAS- 
351 regulated genes was obvious at all experimental time points, and was independent of the plant

352 part under study (i.e., roots vs. shoots) and the direction of regulation (Supplementary Fig.

353 S3).

354 In addition to the ferrome, Fe-responsive genes involved in glycolysis (75 out of a

355 total of 133), the TCA cycle (34/63), JA-signalling (25/36), transcriptional regulation

356 (432/1717), and pre-mRNA splicing (163/396) were analysed. This comparison revealed

357 pronounced differences in splice site strength and intron number among the various groups

358 that appear to be typical of a particular process. When compared with all Fe-responsive

359 entities $(n=9,190)$, genes from the ferrome group, JA-related genes, and genes encoding

360 transcription factors showed a lower-than-average number of introns and a higher-than-

361 average ASS, while genes related to glycolysis, the TCA cycle, and splicing-related genes

362 exhibited an opposite pattern (Fig. 10).

363 Separating exclusively DE- or DAS-regulated genes revealed pronounced differences

364 between the two groups, with DE-regulated genes exhibiting much stronger splice sites and

365 lower intron numbers (Fig. 8b). Strikingly, DAS-regulated transcription factors harbour

366 features of DE-controlled genes, possibly reflecting an evolutionary trend of this group

367 towards this type of regulation. It is further interesting to note that - while containing

368 transcription factors and metabolic genes to almost equal portions - ferrome genes behaved

369 similar to genes encoding transcription factors, suggesting that the massive changes in

370 abundance in response to environmental cues necessitates this type of regulation in most

371 genes in these two groups to allow for an adequate and efficient response to environmental

372 cues.

373 Although promoter length and the number of TFBSs do not seem to affect the mode

374 of gene regulation, these parameters can be supportive to or even critical for gene expression.

375 For example, in yeast, promoters of stress-responsive genes were found to be longer than 
376 those of housekeeping genes ${ }^{51}$, suggesting that such responsiveness requires a more elaborate

377 interaction between trans-acting factors and cis-regulatory elements. In the present study, the

378 average promoter length (1,595 bp) and the average number of TFBSs (33.5) of all 9,190 Fe-

379 responsive genes were not significantly different from those determined for genes of the

380 categories ferrome, glycolysis, and TCA cycle, but slightly higher (38.1) for genes encoding

381 transcription factors (Fig. 9). Crucially, splicing-related genes had on average significantly

382 shorter promotors (1,290 bp) and less TFBSs (27.5), indicating a trend against regulation by

383 DE of these genes. Another deviation from the overall average was observed for JA-related

384 genes, which had longer promoters and more TFBSs (Fig. 11). The promoter architecture of

385 JA-related genes may reflect the extent of recruitment by different signalling pathways. For

386 example, genes encoding enzymes mediating the first steps of JA synthesis (i.e., LOX2,

$387 L O X 3, L O X 4, A O S$, and $A O C 3$ ) had an average promotor length of 2,764 bp and 63.6 TFBSs,

388 almost twice of the average of Fe-responsive genes. JA synthesis is important for a plenitude

389 of processes, which is possibly mirrored by a large number of regulatory cis-consensus

390 motifs on the promotors of these genes. By contrast, clade Ib bHLH proteins, which are

391 highly responsive to the Fe regime but have not been associated with other responses, have

392 short promotors (on average 918 bp) and relatively few TFBSs (24.5). Collectively, the data

393 suggest that a particular process is prone to a certain type of gene regulation, which is, by an

394 appreciable extent, hardwired by the structure of the genes involved in this process.

396 Discussion

397 Multi-layered gene control orchestrates the acclimation to Fe deficiency

398 The current survey shows that short-term exposure to Fe deficiency is sufficient to trigger

399 profound changes in mRNA abundance and AS patterns, inducing a series of responses that

400 act in concert to recalibrate cellular Fe homeostasis. These responses comprise alterations in 
401 transport processes, secondary metabolism, redox and $\mathrm{pH}$ balance, hormone signalling, and

402 central carbon metabolism, and are simultaneously induced in roots and shoots. A less well-

403 explored component Fe deficiency response is the sharply timed induction of JA biosynthesis.

404 Similar to what we describe here for the strategy I plants Arabidopsis, short-term activation

405 of JA biosynthesis was reported for the strategy II plant rice ${ }^{52}$, suggesting that expression of

406 JA-related genes constitutes a general, conserved mechanism across land plants. The reasons

407 for this transient upregulation of JA synthesis are yet to be elucidated. Activation of JA

408 signalling by exogenously supplied methyl JA was shown to affect the assembly of the

409 microbial community in the rhizosphere, possibly by altering the composition of root

410 exudates $^{53}$. In addition, JA determines the compatibility between host and beneficial

411 microbes ${ }^{54,55}$, which, in turn, may positively affect coumarin-mediated Fe uptake ${ }^{56}$. An

412 alternative explanation can be inferred from a study on Arabidopsis plants exposed to

413 oxygen deficiency. Here, a similar boost in JA production was observed in roots after a 6-

414 hour period of oxygen depletion and suggested to trigger repression of root meristem

415 regulators to avoid energy exhaustion when respiration is compromised ${ }^{57}$, a scenario which

416 may also apply to Fe-deficient plants.

417 An underappreciated response to Fe deficiency is the reprogramming of large parts of

418 the central carbon metabolism to counteract imbalances resulting from reduced respiration,

419 decreased activity of Fe-containing enzymes, and shifts in intracellular redox and $\mathrm{pH}$

420 homeostasis. Rerouting central carbon metabolism to prioritize Fe uptake under conditions of

421 Fe deficiency was observed in soil bacteria ${ }^{58}$ and eukaryotic systems such as human

422 macrophages ${ }^{59}$ and yeast ${ }^{15,60,61}$, suggesting that alterations in primary metabolism represent a

423 conserved concept across organisms. Notably, the priority for anaerobic glycolysis, inhibition

424 of PK activity, and the truncated TCA cycle observed in Fe-deficient plant cell resembles the

425 metabolism of cancer cells ${ }^{62}$, further underlining the plasticity of central carbon metabolism 
426 and its role in the adaptation to a given set of conditions. Since many of the observed

427 perturbations such as, for example, the accumulation of pyruvate or citrate are undetectable

428 or at least not pronounced within the first 12 hours of Fe deficiency, it can be assumed that

429 alterations in gene activity occur both in response to and in anticipation of imbalances caused

430 by discontinued Fe supply.

432 Pyruvate is a central player in the metabolic homeostasis of Fe-deficient plants

433 High pyruvate levels stimulate respiration ${ }^{63}$, a situation which is not desirable under

434 conditions of Fe-deficiency. In roots of Fe-deficient plants, prevention of such build-up is

435 attempted by inhibition of $\mathrm{PK}$ and induction of $\mathrm{ADH}$. $\mathrm{ADH}$ transcript and protein levels were

436 found to increase in Fe-deficient roots of various species ${ }^{7,23,42,44,64,65}$, suggesting that ethanol

437 fermentation is a common feature of the Fe deficiency response. In shoots, however, acetic

438 acid is a more likely product of anaerobic respiration. The different fate of pyruvate in root

439 and leaf cells may be associated with different redox status of root and leaf cells. In roots,

440 reduction of acetaldehyde to ethanol restores the $\mathrm{NAD}^{+}$pool to maintain high glycolysis

441 rates $^{66}$. In Fe-deficient leaf cells, acetate synthesis may recalibrate redox homeostasis, a

442 scenario which was suggested to constitute a critical component of a survival strategy

443 triggered by drought stress and possibly other environmental changes ${ }^{67-69}$. In this strategy,

444 acetate formation via ALDH2B7 is crucial in counteracting oxidative stress by stimulating

445 COI1-dependent jasmonate signalling and subsequent induction of JA-responsive genes ${ }^{69}$.

446 Another peculiarity associated with the metabolism of Fe-deficient root cells is related

447 to the massively increased export of protons, which necessitates strategies to replenish

448 substrate for the $\mathrm{H}^{+}$-ATPases and to prevent excessive alkalisation of the cytosol. The

449 concentration of free cytosolic $\mathrm{H}^{+}$is in the sub-micromolar range ${ }^{70}$ and insufficient to support

450 the high proton fluxes of Fe-deficient root cells. Such compensatory release of protons is 
451 achieved by PPC-mediated carboxylation of PEP or, more precisely, the preceding formation

452 of $\mathrm{HCO}_{3}{ }^{-}$by carbonic anhydrase, which is accompanied by a net production of protons (Fig.

453 11). The product of PEP carboxylation, OAA, was not detectable in roots, indicative of its

454 rapid metabolization to citrate, the level of which was dramatically increased upon Fe

455 starvation. Citrate accumulation in roots of Fe-deficient has been reported for a variety of

456 species with high proton extrusion capacity such as tomato ${ }^{16}$, cucumber $^{71,72}$, sugar beet $^{73}$, and

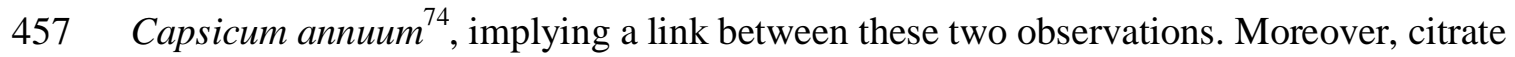

458 levels appear to correlate with PPC activity ${ }^{75}$, making it tempting to speculate that increased

459 proton secretion and subsequent PEP carboxylation are the driving forces for citrate

460 accumulation in Fe-deficient roots. In Arabidopsis, PPC1 was found to be induced in

461 proteomic $^{43}$ and transcriptomic surveys of Fe-deficient roots ${ }^{7,9,43,76,77}$. Moreover,

462 transcriptional induction of PEP carboxylase kinase $(P P C K 1)^{7,9,77,78}$, as well as

463 phosphorylation of PPC1 was observed in Fe-deficient roots ${ }^{23}$, indicating multi-layered and

464 robust regulation of PEP carboxylation in roots upon Fe starvation. It can thus be assumed

465 that citrate accumulation is caused by increased production of OAA via PEP carboxylation

466 and limited metabolization of citrate due to compromised activity of the Fe-sulphur proteins

467 aconitase and succinate dehydrogenase, compromising the completion of the TCA cycle. In

468 Arabidopsis, ATPase-mediated proton export is regulated by FIT $^{79}$, which would imply that

469 citrate accumulation is also dependent on functional FIT protein. In line with this concept, no

470 increased citrate levels upon Fe deficiency were observed in fit mutants ${ }^{80}$. Taken together,

471 these data imply that, with a few exceptions where such changes are induced at the level of

472 transcription (e.g., $P F K, P K, A D H$, and $P P C$ ), DAS appears to represent the main regulon of

473 central carbon metabolism, in particular at early stages of Fe deficiency. This pattern differs

474 from that observed for genes encoding enzymes involved in the production of Fe-mobilizing

475 coumarins, which massively change in abundance both at the transcript and protein levels 
476 (this study) ${ }^{23,81}$. Thus, metabolic processes are not necessarily associated with DAS

477 regulation; it rather appears that the amplitude and specificity of the response (i.e., the direct

478 involvement in Fe uptake) determine the mode of gene regulation.

480 Transcription is biased by but not dependent on genomic features

481 A recent analysis of a suite of AS datasets derived from various eukaryote systems revealed

482 that in addition to or in conjunction with splicing factors, genomic traits may determine the

483 propensity of the mode by which a gene is regulated, suggesting that such features hardwire

484 genes to be controlled by either transcription or alternative splicing ${ }^{30}$. In support of this

485 conception, we show here that in particular the number of introns and the strength of the

486 splicing sites influence the probability of a gene to undergo AS. In contrast to DAS,

487 transcriptional regulation does not appear to be dependent on genomic features. While

488 exclusively DE-regulated genes are strongly biased towards low intron numbers and high

489 splice site strengths, these traits are rather compromising DAS regulation than representing a

490 prerequisite for transcriptional regulation. Transcription appears to be promiscuously

491 employed when pronounced changes in gene activity are required. A representative example

492 is PFK1, which, despite having the architecture typical of DAS-regulated genes and being

493 part of a chiefly DAS-regulated process, is robustly controlled by transcription. It should be

494 noted, however, that transcription-promoting features such as the number of TFBSs may bias

495 the mode of gene regulation towards, or, as in the case of splicing factors, against regulation

496 by DE. The generally observed mutual exclusivity of DE and DAS was not evident in

497 ferrome genes, suggesting that the rapid and highly dynamic responses to the availability of

498 Fe requires a more complex regulation to avoid extreme depletion of or sudden overload with

499 Fe. Thus, under a certain set of conditions, the two mechanisms appear to collaborate to 
500 regulate gene activity. Mostly, but not always, such cooperative regulation occurs anti-

501 directionally, suggesting that DAS is fine-tuning translation efficiency.

502

\section{Conclusions}

504 Collectively, our data show that DE and DAS are co-operative mechanisms that jointly, but

505 mostly autonomously, govern gene activity in a precisely timed pattern in response to

506 environmental signals. The current survey further revealed components of the Fe deficiency

507 response that are either not evident under steady-state conditions (i.e., JA signalling), or not

508 sufficiently mirrored at the transcript level and thus underappreciated in transcriptomic

509 studies (i.e., alterations in pyruvate metabolism). Our data further show that DAS events

510 triggered by short-term exposure to Fe starvation are in large part congruent with

511 observations derived from proteomic, physiological, and metabolic studies conducted at later

512 stages of Fe deficiency, forerunning subsequent alterations in protein abundance and enzyme

513 activity. AS appears to represent an area of largely unexplored 'dark matter', controlling

514 putatively important responses that may significantly contribute to the pronounced

515 discordance of mRNA and protein expression, a gap that is particularly wide in plants ${ }^{82}$. Thus,

516 DAS can be considered as a major contributor of such discordance and a putative proxy for

517 more robust metabolic or physiological changes. A surprising finding was the strong bias

518 towards the mode of gene regulation posed by genomic features. It seems reasonable to

519 speculate that particular processes are more efficiently regulated by either regulatory mode,

520 depending on the function of the genes and the amplitude of the alterations in gene activity

521 required for adequate acclimation to adverse environmental conditions.

\section{Materials and Methods}

\section{Plant Growth}


525 Seeds of Arabidopsis (Arabidopsis thaliana (L.) Heynh) accession Columbia (Col-0) were

526 obtained from the Arabidopsis Biological Resource Center (Ohio State University). Plants

527 were grown hydroponically in a nutrient solution composed of $5 \mathrm{mM} \mathrm{KNO}_{3}, 2 \mathrm{mM} \mathrm{Ca}\left(\mathrm{NO}_{3}\right)_{2}$,

$5282 \mathrm{mM} \mathrm{MgSO}_{4}, 2.5 \mathrm{mM} \mathrm{KH} \mathrm{PO}_{4}, 14 \mu \mathrm{M} \mathrm{MnCl}_{2}, 70 \mu \mathrm{M} \mathrm{H}_{3} \mathrm{BO}_{3}, 1 \mu \mathrm{M} \mathrm{ZnSO}, 0.5 \mu \mathrm{M} \mathrm{CuSO}_{4}$,

$5290.2 \mu \mathrm{M} \mathrm{Na}_{2} \mathrm{MoO}_{4}, 0.01 \mu \mathrm{M} \mathrm{CoCl}_{2}, 40 \mu \mathrm{M}$ Fe-EDTA, and $4.7 \mathrm{mM}$ MES buffer (pH 5.5).

530 Seeds were infiltrated with distilled $\mathrm{H}_{2} \mathrm{O}$ for 3 days in dark in $4^{\circ} \mathrm{C}$ before being transferred to

531 the hydroponic system and then grown in a growth chamber at $21^{\circ} \mathrm{C}$ under continuous

532 illumination $\left(70 \mu \mathrm{mol} \mathrm{m} \mathrm{m}^{-2} \mathrm{~s}^{-1}\right)$. After $16 \mathrm{~d}$ of pre-cultivation, plants were transferred to fresh

533 nutrient solution with either $40 \mu \mathrm{m}$ Fe-EDTA (+Fe plants for control) or no Fe with $100 \mu \mathrm{M}$

534 3-(2-pyridyl)-5,6-diphenyl-1,2,4-triazine sulfonate (-Fe plants) for 0.5-, 6-, and 12-hours

535 treatment for RNA-seq analysis, and 1-3 days -Fe treatment for further RT-qPCR

536 experiments or Ultra-High Performance Liquid Chromatography (UHPLC) analysis. At the

537 end of the treatment, root and shoot were collected and stored at $-80^{\circ} \mathrm{C}$.

\section{RNA-seq and definition of DEGs}

540 Total RNA was extracted from approximately $100 \mathrm{mg}$ of Arabidopsis roots or shoots using

541 the RNeasy Plant Mini Kit (Qiagen, Cat. No. 74904). RNA samples were treated with

542 DNaseI (Qiagen, Cat. No. 79254) to remove DNA. RNA concentration was determined with

543 a NanoDrop ND-1000 UV-Vis spectrophotometer (NanoDrop Technologies). For preparing

544 RNA-seq libraries, mRNA molecules with poly-A tails were purified using poly-T oligo-

545 attached magnetic beads.

546 The first-strand cDNA was synthesized by the use of dNTP (dUTP replaced by dTTP), buffer,

547 RNaseH, and DNA polymerase I. cDNA was purified using a Purification Kit (Qiagen)

548 followed by performing end repair and A-tailing. The sample was then treated with

549 USERTM (Uracil-Specific Excision Reagent) enzyme to digest the antisense strand DNA 
550 follow by PCR reaction. After these procedures, the library could be sequenced using the

551 Illumina HiSeq 4000 platform. The first step in the trim process was the conversion of the

552 quality score (Q) to error probability. Next, for every base a new value was calculated; 0.05 -

553 error probability. This value is negative for low quality bases, where the error probability is

554 high. For every base, we calculated the running sum of this value. If the sum dropped below

555 zero, it was set to zero. The part of the sequence to be retained is between the first positive

556 value of the running sum and the highest value of the running sum. Everything before and

557 after this region was trimmed off. In addition, reads shorter than 35 bp were discarded. A

558 total of 66 to 87 million reads were obtained from Illumina sequencing for the various

559 libraries (Supplementary Table S1). Reads were aligned to the TAIR10 transcriptome using

560 Bowtie $2^{83}$, and only alignments of read pairs that mapped to the same transcripts were

561 accepted. The remaining reads were mapped to the TAIR10 genome directly using the BLAT

562 program $^{84}$ with default parameters. Alignments with a minimum $95 \%$ identity for each read

563 were considered for mapping but only the alignment with the highest identity were accepted.

564 Read counts were computed using the RACKJ software package

565 (http://rackj.sourceforge.net/), normalized using the Trimmed Mean of M-values (TMM)

566 method $^{85}$, and transformed into log-count-per-million (logCPM) using the voom method ${ }^{86}$.

567 Adjusted RPKM values (Reads Per Kilobase of exon Model per million mapped reads ${ }^{87}$ )

568 were computed based on $\log$ CPMs and gene model lengths. For two given samples, the

569 RPKM values of the genes was compared using $t$-tests, and a gene was identified as

570 differentially expressed if the corresponding $P$ value was less than or equal to 0.05 and the

571 fold-change was greater than 2 at each time point. Only genes with relevant expression levels

572 (RPKM > the square root of the mean expression value of the whole dataset) were considered.

\section{$574 \quad$ Alternative splicing analysis}


575 Alternative splicing events were identified as described previously ${ }^{88}$ using the RACKJ

576 software. Three types of alternative splicing were considered, IR, DA, and ES. For detecting

577 IR events, the IR ratio was computed as the average read depth of its intron divided by the

578 average read depth of the neighboring exons, and the IR ratios of three -Fe replicates were

579 compared to those of the controls $(+\mathrm{Fe})$ using $t$-test. Similarly, to detect alternative

580 donor/acceptor or exon skipping events, signals representing AS events (read counts skipping

581 exons, and read counts covering the same splicing junctions) were divided by gene

582 expression levels as background. $T$-tests were performed on the obtained ratios to compare

583 samples from treated plants against control samples. Changes of relative expression levels of

584 AS events were inferred using a $t$-test $\mathrm{P}$ value $<0.05$ with a fold-change $>2$.

\section{$586 \quad$ RT-qPCR}

587 Samples were frozen in liquid nitrogen at the end of the experimental period and stored at -

$58880^{\circ} \mathrm{C}$. Total RNA was extracted using the RNeasy Mini Kit (Qiagen) and treated with DNase

589 using the TURBO DNA-free kit (Ambion). Three $\mu \mathrm{g}$ of total RNA per sample was used for

590 obtaining cDNAs. First-strand cDNA was synthesized using oligo(dT) primer and the

591 SupersCript ${ }^{\mathrm{TM}}$ III First-Strand Synthesis System (Invitrogen, Cat. No. 18080) for RT-qPCR.

592 The resulting single-stranded complementary cDNAs were then used as a template in real-

593 time RT-PCR assay. RT-qPCRs were carried out with gene-specific primers listed in Table

594 S6, and SYBR ${ }^{\mathrm{TM}}$ Green PCR Master Mix (Applied Biosystems, Cat. No. 4367659) according

595 to the manufacturer's instructions using a QuantStudio 12K Flex Real-Time PCR System.

596 Three independent replicates were performed for each sample. The $\Delta \Delta \mathrm{C}_{\mathrm{T}}$ method was used to

597 determine the relative gene expression ${ }^{89}$, with the expression of elongation factor 1 alpha

598 (EF1 $\alpha$; At5g60390) used as an internal control. 


\section{Genomic analyses}

601 Five' and 3' splicing site strength scores and event information were downloaded from the

602 PastDB dabase ${ }^{30}$. An inhouse Perl script was developed to associate AS events of PastDB

603 with the accession number of genes from the TAIR10 annotation. The information on

604 transcription factor binding sites and promoter lengths was downloaded from the Arabidopsis

605 thaliana cis-regulatory database (AtcisDB) database on the Arabidopsis Gene Regulatory

606 Information Server (AGRIS) ${ }^{90}$.

607

608 UHPLC-MS analysis

609 Approximately $100 \mathrm{mg}$ plant tissues were harvested, extracted in $1.5 \mathrm{ml}$ of a solution of 375

$610 \mu \mathrm{ld} \mathrm{d}_{2} \mathrm{O}, 750 \mu \mathrm{l}$ methanol, $375 \mu \mathrm{l}$ chloroform, and an internal standard (Citrate-2,2,4,4- $\mathrm{d}_{4}$,

611 CDN ISOTOPES, Cat. No. 147664-83-3) was added to final concentration of $10 \mu \mathrm{M}$ for each

612 sample. The supernatant was separated by quick spin-down at $3,000 \mathrm{xg}$, incubated at $-20{ }^{\circ} \mathrm{C}$

613 for $30 \mathrm{~min}$, and centrifugated at 3,000xg for $10 \mathrm{~min}$ at $4{ }^{\circ} \mathrm{C}$. Obtained supernatant was mixed

614 by vortexing with $375 \mu$ chloroform of chloroform to remove pigments. Colorless

615 supernatant was dried in a SpeedVac, resuspended in 50\% methanol, and kept under $-80{ }^{\circ} \mathrm{C}$.

616 A Vanquish ${ }^{\mathrm{TM}}$ Horizon UHPLC System (Thermo Scientific) coupled to an Orbitrap Fusion

617 Lumos (Thermo Scientific) mass spectrometer was used for the LC-MS analysis. The

618 chromatographic separation for samples was carried out on Atlantis Premier BEH C18 AX

619 VanGuard FIT Column, $1.7 \mu \mathrm{m}, 2.1$ x $100 \mathrm{~mm}$ column (Waters). The column was

620 maintained at a temperature of $30^{\circ} \mathrm{C}$ and $1 \mu \mathrm{L}$ sample were injected per run. The mobile

621 phase A was $2 \%$ Acetonitrile $0.1 \% \mathrm{v} / \mathrm{v}$ formic acid in water and mobile phase B was $40 \% \mathrm{v} / \mathrm{v}$

622 acetonitrile with $20 \mathrm{mM}$ ammonium formate $\mathrm{pH}$ 3.0. The gradient elution with a flow rate

$6230.4 \mathrm{~mL} / \mathrm{min}$ was performed with a total analysis time of $11 \mathrm{~min}$. The gradient included $0.5 \%$

$624 \mathrm{~B}$ at $0 \square \min$, a hold at $0.5 \% \mathrm{~B}$ until $2 \square \min , 99.5 \% \mathrm{~B}$ at $6 \square \mathrm{min}$, a hold at $99.5 \% \mathrm{~B}$ until 
$6258 \square \min , 0.5 \% \mathrm{~B}$ at $8.5 \square \mathrm{min}$, and a hold at $0.5 \% \mathrm{~B}$ until $11 \square \mathrm{min}$. General instrumental

626 conditions were RF lens $60 \%$; sheath gas, auxiliary gas, and sweep gas of 50, 10, and 3

627 arbitrary units, respectively; ion transfer tube temperature of $325^{\circ} \mathrm{C}$; vaporizer temperature

628 of $350{ }^{\circ} \mathrm{C}$; and spray voltage of $3500 \mathrm{~V}$ for negative mode. For analysis, a full MS scan mode

629 with a scan range $m / z 50$ to 400 , resolution 30,000, AGC target $4 \mathrm{e} 5$ and a maximum injection

630 time $50 \mathrm{~ms}$ was applied. The Xcalibur 4.1 software (Thermo Scientific) was used for the data

631 processing.

632

633 Acknowledgements

634 We thank Dr. Yuki Nakamura (IPMB, Academia Sinica) for kindly providing chemicals for

635 UHPLC standard and Shou-Jen Chou in the Genomic Technology Core Facility at IPMB for

636 preparing libraries for RNA-seq analysis. We also thank Mei-Jane Fang at the Genomic

637 Technology Core Facility at the Institute of Plant and Microbial Biology for using of the

638 QuantStudio 12K Flex Real-Time PCR System, and Yu-Ching Wu for the UHPLC-MS

639 analysis, which was supported by the Academia Sinica Metabolomics Core Facility at the

640 Agricultural Biotechnology Research Center of Academia Sinica, supported by Academia

641 Sinica Core Facility and Innovative Instrument Project (AS-CFII-108-108).

643 Author contributions

644 E.J.H. and W.S. designed the research; E.J.H. performed the experiments; E.J.H., W.D.L., 645 and W.S. analysed the data; W.S. and E.J.H., wrote the manuscript.

\section{Conflict of interest}

648 The authors declare no conflict of interest. 


\section{Data availability}

651 The RNA-seq data have been deposited at NCBI under the accession number PRJNA759647

652 Reviewer link:

653 https://dataview.ncbi.nlm.nih.gov/object/PRJNA759647?reviewer=itt5n0

654 pfr6mmqho1akj54r5g2k

655

656

\section{References}

1. Kobayashi, T. et al. OsbHLH058 and OsbHLH059 transcription factors positively regulate iron deficiency responses in rice. Plant Mol. Biol. 101, 471-486 (2019).

2. Gao, F. \& Dubos, C. Transcriptional integration of plant responses to iron availability. $J$. Exp. Bot. 72, 2056-2070 (2021).

3. Ro $\square$ mheld, V. \& Marschner, H. Mechanism of iron uptake by peanut plants: I. FeIII reduction, chelate splitting, and release of phenolics. Plant Physiol. 71, 949-954 (1983).

4. Eide, D., Broderius, M., Fett, J. \& Guerinot, M. L. A novel iron-regulated metal transporter from plants identified by functional expression in yeast. Proc. Natl. Acad. Sci. U.S.A. 93, 5624-5628 (1996).

5. Robinson, N. J., Procter, C. M., Connolly, E. L. \& Guerinot, M. L. A ferric-chelate reductase for iron uptake from soils. Nature 397, 694-697 (1999).

6. Santi, S. \& Schmidt, W. Dissecting iron deficiency $\square$ induced proton extrusion in Arabidopsis roots. New Phytol. 183, 1072-1084 (2009).

7. Rodríguez-Celma, J. et al. Mutually exclusive alterations in secondary metabolism are critical for the uptake of insoluble iron compounds by Arabidopsis and Medicago truncatula. Plant Physiol. 162, 1473-1485 (2013a).

8. Schmid, N. B. et al. Feruloyl-CoA 6'-Hydroxylase1-dependent coumarins mediate iron acquisition from alkaline substrates in Arabidopsis. Plant Physiol. 164, 160-172 (2014).

9. Grillet, L., Lan, P., Li, W., Mokkapati, G. \& Schmidt, W. IRON MAN is a ubiquitous family of peptides that control iron transport in plants. Nat. Plants 4, 953-963 (2018).

10. Gao, F., Robe, K., Gaymard, F., Izquierdo, E. \& Dubos, C. The transcriptional control of iron homeostasis in plants: a tale of bHLH transcription factors? Front. Plant Sci. 10, 6 (2019).

11. Kim, S. A., LaCroix, I. S., Gerber, S. A. \& Guerinot, M. L. The iron deficiency response in Arabidopsis thaliana requires the phosphorylated transcription factor URI. Proc. Natl. Acad. Sci. U.S.A. 116, 24933-24942 (2019).

12. Lei, R. et al. bHLH121 functions as a direct link that facilitates the activation of FIT by bHLH IVc transcription factors for maintaining Fe homeostasis in Arabidopsis. Mol. Plant 13, 634-649 (2020).

13. Oexle, H., Gnaiger, E. \& Weiss, G. Iron-dependent changes in cellular energy metabolism: influence on citric acid cycle and oxidative phosphorylation. Biochim. Biophys. Acta Bioenerg. 1413, 99-107 (1999).

14. Pereira, M. et al. Acute iron deprivation reprograms human macrophage metabolism and reduces inflammation in vivo. Cell Rep. 28, 498-511. e5 (2019).

15. Shakoury-Elizeh, M. et al. Metabolic response to iron deficiency in Saccharomyces cerevisiae. J. Biol. Chem. 285, 14823-14833 (2010). 
16. López-Millán, A. F., Morales, F., Gogorcena, Y., Abadía, A. \& Abadía, J. Metabolic responses in iron deficient tomato plants. J. Plant Physiol. 166, 375-384 (2009).

17. Zocchi, G. in Iron nutrition in plants and rhizospheric microorganisms. 359-370 (Springer, 2006).

18. Zocchi, G., De Nisi, P., Dell'Orto, M., Espen, L. \& Gallina, P. M. Iron deficiency differently affects metabolic responses in soybean roots. J. Exp. Bot. 58, 993-1000 (2007).

19. Rellán-Álvarez, R. et al. Identification of a tri-iron (III), tri-citrate complex in the xylem sap of iron-deficient tomato resupplied with iron: new insights into plant iron longdistance transport. Plant Cell Physiol. 51, 91-102 (2010).

20. Mai, H. \& Bauer, P. From the proteomic point of view: Integration of adaptive changes to iron deficiency in plants. Curr. Plant Biol. 5, 45-56 (2016).

21. Espen, L., Dell'Orto, M., De Nisi, P. \& Zocchi, G. Metabolic responses in cucumber (Cucumis sativus L.) roots under Fe-deficiency: a 31 P-nuclear magnetic resonance invivo study. Planta 210, 985-992 (2000).

22. Herbik, A. et al. Iron and copper nutrition-dependent changes in protein expression in a tomato wild type and the nicotianamine-free mutant chloronerva. Plant Physiol. 111, 533-540 (1996).

23. Lan, P. et al. iTRAQ protein profile analysis of Arabidopsis roots reveals new aspects critical for iron homeostasis. Plant Physiol. 155, 821-834 (2011).

24. Marquez, Y., Brown, J. W., Simpson, C., Barta, A. \& Kalyna, M. Transcriptome survey reveals increased complexity of the alternative splicing landscape in Arabidopsis. Genome Res. 22, 1184-1195 (2012).

25. Reddy, A. S., Rogers, M. F., Richardson, D. N., Hamilton, M. \& Ben-Hur, A. Deciphering the plant splicing code: experimental and computational approaches for predicting alternative splicing and splicing regulatory elements. Front. Plant Sci. 3, 18 (2012).

26. Keren, H., Lev-Maor, G. \& Ast, G. Alternative splicing and evolution: diversification, exon definition and function. Nat. Rev. Genet, 11, 345-355 (2010).

27. Kalyna, M. et al. Alternative splicing and nonsense-mediated decay modulate expression of important regulatory genes in Arabidopsis. Nucleic Acids Res. 40, 2454-2469 (2012).

28. $\mathrm{Li}, \mathrm{H}$. et al. Histone acetylation associated up-regulation of the cell wall related genes is involved in salt stress induced maize root swelling. BMC Plant Biol. 14, 1-14 (2014).

29. Chaudhary, S., Jabre, I., Reddy, A. S., Staiger, D. \& Syed, N. H. Perspective on alternative splicing and proteome complexity in plants. Trends Plant Sci. 24, 496-506 (2019).

30. Martín, G., Márquez, Y., Mantica, F., Duque, P. \& Irimia, M. Alternative splicing landscapes in Arabidopsis thaliana across tissues and stress conditions highlight major functional differences with animals. Genome Biol. 22, 1-26 (2021).

31. Fu, X. \& Ares, M. Context-dependent control of alternative splicing by RNA-binding proteins. Nat. Rev. Genet. 15, 689-701 (2014).

32. Calixto, C. P. et al. Rapid and dynamic alternative splicing impacts the Arabidopsis cold response transcriptome. Plant Cell 30, 1424-1444 (2018).

33. Dikaya, V. et al. Insights into the role of alternative splicing in plant temperature response. J. Exp. Bot. (2021).

34. Morton, M., AlTamimi, N., Butt, H., Reddy, A. S. \& Mahfouz, M. Serine/Arginine-rich protein family of splicing regulators: New approaches to study splice isoform functions. Plant Sci. 283, 127-134 (2019).

35. Laloum, T., Martín, G. \& Duque, P. Alternative splicing control of abiotic stress responses. Trends Plant Sci. 23, 140-150 (2018). 
36. Luco, R. F., Allo, M., Schor, I. E., Kornblihtt, A. R. \& Misteli, T. Epigenetics in alternative pre-mRNA splicing. Cell 144, 16-26 (2011).

37. Pajoro, A., Severing, E., Angenent, G. \& Immink, R. Histone H3 lysine 36 methylation affects temperature-induced alternative splicing and flowering in plants. Genome Biol. 18, 1-12 (2017).

38. Jabre, I. et al. Differential nucleosome occupancy modulates alternative splicing in Arabidopsis thaliana. New Phytol. 229, 1937-1945 (2021).

39. Li, W., Lin, W., Ray, P., Lan, P. \& Schmidt, W. Genome-wide detection of conditionsensitive alternative splicing in Arabidopsis roots. Plant Physiol. 162, 1750-1763 (2013).

40. Palmer, C. M., Hindt, M. N., Schmidt, H., Clemens, S. \& Guerinot, M. L. MYB10 and MYB72 are required for growth under iron-limiting conditions. PLoS Genet. 9, e1003953 (2013).

41. Lan, P., Li, W. \& Schmidt, W. Complementary proteome and transcriptome profiling in phosphate-deficient Arabidopsis roots reveals multiple levels of gene regulation. Mol. Cell. Proteomics 11, 1156-1166 (2012).

42. Donnini, S. et al. Proteomic characterization of iron deficiency responses in Cucumis sativus L. roots. BMC Plant Biol. 10, 1-15 (2010).

43. Pan, I. C. et al. Post-transcriptional coordination of the Arabidopsis iron deficiency response is partially dependent on the E3 Ligases RING DOMAIN LIGASE1 (RGLG1) and RING DOMAIN LIGASE2 (RGLG2). Mol. Cell. Proteomics 14, 2733-2752 (2015).

44. Thimm, O., Essigmann, B., Kloska, S., Altmann, T. \& Buckhout, T. J. Response of Arabidopsis to iron deficiency stress as revealed by microarray analysis. Plant Physiol. 127, 1030-1043 (2001).

45. Plaxton, W. C. The organization and regulation of plant glycolysis. Annu. Rev. Plant Biol. 47, 185-214 (1996).

46. O'Leary, B. \& Plaxton, W. C. Multifaceted functions of post-translational enzyme modifications in the control of plant glycolysis. Curr. Opin. Plant Biol. 55, 28-37 (2020).

47. Plaxton, W. C. \& Podestá, F. E. The functional organization and control of plant respiration. Crit. Rev. Plant Sci. 25, 159-198 (2006).

48. Rodríguez-Celma, J. et al. The transcriptional response of Arabidopsis leaves to Fe deficiency. Front. Plant Sci. 4, 276 (2013).

49. Schmidt, W. \& Buckhout, T. J. A hitchhiker's guide to the Arabidopsis ferrome. Plant Physiol. Biochem. 49, 462-470 (2011).

50. Stringlis, I. A. et al. MYB72-dependent coumarin exudation shapes root microbiome assembly to promote plant health. Proc. Natl. Acad. Sci. U.S.A. 115, E5213-E5222 (2018).

51. Kristiansson, E., Thorsen, M., Tamás, M. J. \& Nerman, O. Evolutionary forces act on promoter length: identification of enriched cis-regulatory elements. Mol. Biol. Evol. 26, 1299-1307 (2009).

52. Kobayashi, T. et al. Jasmonate signaling is activated in the very early stages of iron deficiency responses in rice roots. Plant Mol. Biol. 91, 533-547 (2016).

53. Carvalhais, L. C. et al. Linking jasmonic acid signaling, root exudates, and rhizosphere microbiomes. Mol. Plant-Microbe Interact. 28, 1049-1058 (2015).

54. Jacobs, S. et al. Broad-spectrum suppression of innate immunity is required for colonization of Arabidopsis roots by the fungus Piriformospora indica. Plant Physiol. 156, 726-740 (2011).

55. Stringlis, I. A., De Jonge, R. \& Pieterse, C. M. The age of coumarins in plant-microbe interactions. Plant Cell Physiol. 60, 1405-1419 (2019).

56. Harbort, C. J. et al. Root-secreted coumarins and the microbiota interact to improve iron nutrition in Arabidopsis. Cell Host Microbe 28, 825-837. e6 (2020). 
57. Shukla, V. et al. Jasmonate signalling contributes to primary root inhibition upon oxygen deficiency in Arabidopsis thaliana. Plants 9, 1046 (2020).

58. Mendonca, C. M. et al. Hierarchical routing in carbon metabolism favors iron-scavenging strategy in iron-deficient soil Pseudomonas species. Proc. Natl. Acad. Sci. U.S.A. 117, 32358-32369 (2020).

59. Pereira, M. et al. Acute iron deprivation reprograms human macrophage metabolism and reduces inflammation in vivo. Cell Reports 28, 498-511 (2019).

60. Puig, S., Askeland, E., Thiele, D. J. Coordinated remodeling of cellular metabolism during iron deficiency through targeted mRNA degradation. Cell 120, 99-110 (2005).

61. Chen, Y., Li, F., Mao, J., Chen, Y. \& Nielsen, J. Yeast optimizes metal utilization based on metabolic network and enzyme kinetics. Proc. Natl. Acad. Sci. U.S.A. 118, 10.1073/pnas.2020154118 (2021).

62. Dayton, T. L. et al. Germline loss of PKM2 promotes metabolic distress and hepatocellular carcinoma. Genes Dev. 30, 1020-1033 (2016).

63. Zabalza, A. et al. Regulation of respiration and fermentation to control the plant internal oxygen concentration. Plant Physiol. 149, 1087-1098 (2009).

64. Mai, H. et al. Iron and FER $\square$ LIKE IRON DEFICIENCY $\square$ INDUCED TRANSCRIPTION FACTOR $\square$ dependent regulation of proteins and genes in Arabidopsis thaliana roots. Proteomics 15, 3030-3047 (2015).

65. Rodríguez-Celma, J. et al. Characterization of flavins in roots of Fe-deficient strategy I plants, with a focus on Medicago truncatula. Plant Cell Physiol. 52, 2173-2189 (2011).

66. Bui, L. T. et al. Conservation of ethanol fermentation and its regulation in land plants. $J$. Exp. Bot. 70, 1815-1827 (2019).

67. Kursteiner, O., Dupuis, I. \& Kuhlemeier, C. The pyruvate decarboxylase1 gene of Arabidopsis is required during anoxia but not other environmental stresses. Plant Physiol. 132, 968-978 (2003).

68. Rasheed, S. et al. The modulation of acetic acid pathway genes in Arabidopsis improves survival under drought stress. Sci. Rep. 8, 1-15 (2018).

69. Kim, J. et al. Acetate-mediated novel survival strategy against drought in plants. Nat. Plants 3, 1-7 (2017).

70. Wegner, L. H. et al. Biochemical and biophysical $\mathrm{pH}$ clamp controlling Net $\mathrm{H}$ efflux across the plasma membrane of plant cells. New Phytol. 230, 408-415 (2021).

71. Rabotti, G., De Nisi, P. \& Zocchi, G. Metabolic implications in the biochemical responses to iron deficiency in cucumber (Cucumis sativus L.) roots. Plant Physiol. 107, 1195-1199 (1995).

72. Nisi, P. D. \& Zocchi, G. Phosphoenolpyruvate carboxylase in cucumber (Cucumis sativus L.) roots under iron deficiency: activity and kinetic characterization. J. Exp. Bot. 51, 1903-1909 (2000).

73. López-Millán, A. F., Morales, F., Abadía, A. \& Abadía, J. Effects of iron deficiency on the composition of the leaf apoplastic fluid and xylem sap in sugar beet. Implications for iron and carbon transport. Plant Physiol. 124, 873-884 (2000).

74. Landsberg, E. Function of rhizodermal transfer cells in the Fe stress response mechanism of Capsicum annuum L. Plant Physiol. 82, 511-517 (1986).

75. Abadía, J., López-Millán, A., Rombolà, A. \& Abadía, A. Organic acids and Fe deficiency: a review. Plant Soil 241, 75-86 (2002).

76. Dinneny, J. R. et al. Cell identity mediates the response of Arabidopsis roots to abiotic stress. Science 320, 942-945 (2008).

77. Yang, T. J., Lin, W. \& Schmidt, W. Transcriptional profiling of the Arabidopsis iron deficiency response reveals conserved transition metal homeostasis networks. Plant Physiol. 152, 2130-2141 (2010). 
78. Bailey, K. J., Gray, J. E., Walker, R. P. \& Leegood, R. C. Coordinate regulation of phosphoenolpyruvate carboxylase and phosphoenolpyruvate carboxykinase by light and $\mathrm{CO}_{2}$ during C4 photosynthesis. Plant Physiol. 144, 479-486 (2007).

79. Mai, H., Pateyron, S. \& Bauer, P. Iron homeostasis in Arabidopsis thaliana: transcriptomic analyses reveal novel FIT-regulated genes, iron deficiency marker genes and functional gene networks. BMC Plant Biol. 16, 1-22 (2016).

80. Chutia, R., Scharfenberg, S., Neumann, S., Abel, S. \& Ziegler, J. Modulation of phosphate deficiency-induced metabolic changes by iron availability in Arabidopsis thaliana. Int. J. Mol. Sci. 22, 7609 (2021).

81. Tsai, H. et al. Scopoletin 8-hydroxylase-mediated fraxetin production is crucial for iron mobilization. Plant Physiol. 177, 194-207 (2018).

82. Vélez-Bermúdez, I. C. \& Schmidt, W. The conundrum of discordant protein and mRNA expression. Are plants special? Front. Plant Sci. 5, 619 (2014).

83. Langmead, B. \& Salzberg, S. L. Fast gapped-read alignment with Bowtie 2. Nat. Methods 9, 357-359 (2012).

84. Kent, W. J. BLAT--the BLAST-like alignment tool. Genome Res. 12, 656-664 (2002).

85. Robinson, M. D. \& Oshlack, A. A scaling normalization method for differential expression analysis of RNA-seq data. Genome Biol. 11, 1-9 (2010).

86. Law, C. W., Chen, Y., Shi, W. \& Smyth, G. K. voom: Precision weights unlock linear model analysis tools for RNA-seq read counts. Genome Biol. 15, 1-17 (2014).

87. Mortazavi, A., Williams, B. A., McCue, K., Schaeffer, L. \& Wold, B. Mapping and quantifying mammalian transcriptomes by RNA-Seq. Nat. Methods 5, 621-628 (2008).

88. Kanno, T., Lin, W., Chang, C., Matzke, M. \& Matzke, A. J. A genetic screen identifies PRP18a, a putative second step splicing factor important for alternative splicing and a normal phenotype in Arabidopsis thaliana. G3 8, 1367-1377 (2018).

89. Livak, K. J. \& Schmittgen, T. D. Analysis of relative gene expression data using real-time quantitative PCR and the 2- $\Delta \Delta$ CT method. Methods 25, $402-408$ (2001).

90. Yilmaz, A. et al. AGRIS: the Arabidopsis gene regulatory information server, an update. Nucleic Acids Res. 39, D1118-D1122 (2010).

\section{Figure legends:}

658 Figure 1. Differentially expressed genes (DEGs) and differentially alternatively spliced

659 (DAS) transcripts in response to short-term exposure to Fe deficiency. a) Filters for the

660 identification and numbers of DEGs at the various experimental time points. b) Venn

661 diagrams showing the overlaps of DEGs among the different time points. c) Filters for the

662 identification and numbers of DAS genes. d) Venn diagrams showing the overlaps of DAS

663 genes among the different time points. e) Venn diagrams showing the overlaps of DIR, DDA,

664 and DES genes in Fe-deficient roots. f) Venn diagrams showing the overlaps of DEG and 
665 DAS genes in roots and shoots of Fe-deficient plants. DIR, differential intron retention; DDA,

666 differential donor or acceptor sites; DES, differential exon skipping; FC, foldchange.

667

668 Figure 2. Functions of Fe-responsive genes in Fe uptake and cellular Fe homeostasis. a-d)

669 Cartoon showing DEG and DAS genes in roots (upper panels) and shoots (lower panels) after

$6706 \mathrm{~h}(\mathrm{a}, \mathrm{c})$ and $12 \mathrm{~h} \mathrm{Fe}(\mathrm{b}, \mathrm{d})$ deficiency. For the transcriptionally regulated genes, the direction

671 of regulation is indicated by red and blue arrows for up- and down-regulated genes,

672 respectively. DAS genes are denoted by italics, differentially expressed DAS genes are

673 indicated by italics and bold letters. The direction of DAS regulation is indicated by red

674 (enhanced) or blue (reduced) AS. Detailed explanations of gene functions are given in the

675 text.

676

677 Figure 3. Induction of jasmonate biosynthesis, catabolism, and signalling genes in roots in

678 response to 6 hours Fe deficiency treatment. In plastids, lipid-derived $\alpha$-linolenic acid is

679 converted by LOX, AOS, and AOC into oxophytodienoic acid (OPDA). Following transport

680 into peroxisomes, OPDA is reduced by OPDA REDUCTASE 3 (OPR3) and converted into

681 JA by $\beta$-oxidation. In the cytoplasm, JA is conjugated with amino acids to JA-Ile by JAR1 or

682 to MeJA by JMT. In the endoplasmic reticulum, JA-Ile is degraded to 12OH-JA-Ile and

683 converted to 12-OH-JA via IAR3, ILL5 and ILL6. The latter enzymes can also convert JA-Ile

684 to JA. In the absence of nuclear JA-Ile, expression of JA responsive genes via MYC2 is

685 repressed by JAZ. Upon the entry of JA-Ile into the nucleus, JAZ is degraded via the $26 \mathrm{~S}$

686 proteasome and ultimately triggers transcriptional activation of the target genes. Only genes

687 that were differentially expressed or harboured DAS features in response to Fe starvation are

688 shown.

689 
690 Figure 4. Regulation of glycolytic enzymes and their products by Fe-deficiency. a) DAS

691 regulation of genes encoding glycolytic enzymes in roots. b) Concentrations of glucose-6-

692 phosphate (G6P), fructose-6-phosphate F6P/glucose-1-phosphate (G1P) (G6P and G1P have

693 identical retention times and cannot be distinguished from each other), phosphoenolpyruvate

694 (PEP) and pyruvate in roots and shoots 12 hours and 3 days after transfer to Fe-deplete media.

695 c-e) qRT-PCR analysis of PFK (c), PK (d; At5g08570) and bHLH38 (e). Asterisks indicate

696 significant differences from the wild type in each treatment: *, $P<0.05 ; * *, P<0.01 ; * * *, P$

$697<0.001$. C, control.

699 Figure 5. Pyruvate metabolism in roots and shoots of Fe-deficient plants. a, b) Fe-responsive

700 enzymes in roots (a) and shoots (b). Only genes that were differentially expressed or

701 harboured DAS (DIR or DDA) features in response to Fe starvation are shown. See text for

702 details.

703

704 Figure 6. Correlation between DE and DAS of Fe-responsive genes. a) DE versus DIR. b)

705 DE versus DDA.

707 Figure 7. Relative contribution of DE and DAS in the regulation of Fe-responsive genes. a)

708 Time-course of DE- and DAS-regulated genes in roots and shoots in response to Fe

709 deficiency. b) DE and DAS regulation in various categories of ferrome genes after short-tern

710 exposure (left panel) and three days after transfer to Fe-deficient conditions (right panel).

711 Short-term data are pooled from three experimental time points $(0.5,6$, and $12 \mathrm{~h})$. Long-term

712 data are taken from a previous study (Li et al., 2014). c) DE and DAS regulation of genes

713 involved in various processes after short-term exposure to Fe-deficient conditions. d) DE and

714 DAS regulation after long-term exposure (3 d) to Fe-deficient conditions. 
716 Figure 8. Correlation of architectural traits of Fe-responsive genes with the mode of gene

717 regulation. a, b) Intron number (blue bars) and average minimum 5' and 3' splice site (SS)

718 strength (red bars) of all Fe-responsive genes (a), and DEGs, DAS genes, and genes that are

719 regulated by both DE and DAS (b). c, d) Correlation between the number of transcription

720 factor binding sites (TFBSs) and promotor length of all Fe-responsive genes (a), and DEGs,

721 DAS genes, and genes that are regulated by both DE and DAS (b).

723 Figure 9. Correlation of architectural traits of individual ferrome gene with the mode of gene

724 regulation. a-c) Intron number (blue bars) and average minimum 5' and 3' splice site (SS)

725 strength (red bars) of DEGs (a) DAS genes (b), and genes that are regulated by both DE and

726 DAS (c).

728 Figure 10. Genomic features of Fe-responsive genes involved in various processes. a)

729 Average minimum strengths of 5' and 3' splice sites. b) Intron number. c) Transcription

730 factor binding sites (TFBSs). d) Promoter length. Box plot shows the median (line) and the

731 average (x) for genes in roots (a) and shoots (b). Significant differences were detected using

732 two-way ANOVA with Tukey's multiple comparison test, $P<0.001$.

733

734 Figure 11. Summary of the changes in central carbon metabolism in roots of Fe deficient

735 plants. Carbon flux through the glycolytic pathway is increased by transcriptional

736 upregulation of PFK1. Pyruvate accumulation caused by a truncated TCA cycle is avoided by

737 downregulation of PK activity by DAS and DE and increased ethanolic respiration via PDC1

738 and $\mathrm{ADH} 1$, which are regulated by DAS and DE. This reaction regenerates the $\mathrm{NAD}^{+}$pool

739 for continued glycolytic flux. Acetate respiration, which is prioritized in shoots, is repressed 
740 by DAS of $A L D H 7 B 4$. Increased net proton secretion by P-type ATPases is counteracted by

741 the formation of $\mathrm{HCO}_{3}{ }^{-}$and subsequent carboxylation of phosphoenolpyruvate (PEP),

742 yielding oxaloacetate (OAA), which is converted to citrate. DAS genes are denoted by italics,

743 genes regulated by both DE and DAS are indicated by italics and bold letters. Enzymes that

744 are not differentially expressed are depicted in grey. The direction of DAS regulation is

745 indicated in red (enhanced) or blue (reduced) letters. 
(which was not certified by peer review) is the author/funder, who has granted bioRxiv a license to display the preprint in perpetuity. It is

Roots

\section{DEG}

Total genes detected

$$
\begin{array}{ccccccc}
0.5 \mathrm{~h} & 6 \mathrm{~h} & 12 \mathrm{~h} & 0.5 \mathrm{~h} & 6 \mathrm{~h} & 12 \mathrm{~h} \\
27,148 & 24,079 & 27,080 & 27,613 & 27,437 & 27,185
\end{array}
$$$$
\begin{gathered}
\text { Background subtraction } \\
>m^{\wedge}(1 / 2)
\end{gathered}
$$$$
15,697 \quad 15,717 \quad 15,521
$$

$14,972 \quad 15,119 \quad 15,181$
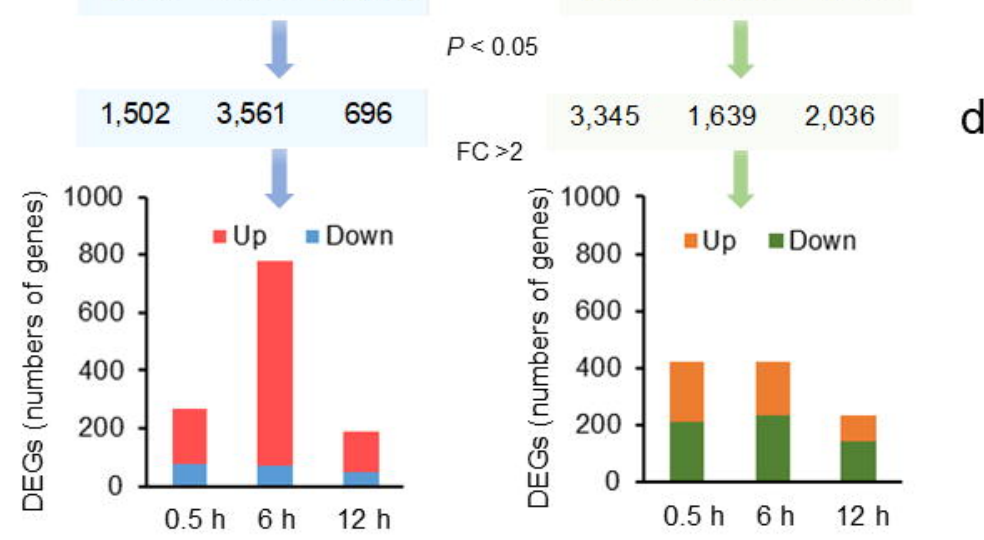

b

Total AS feature

Number of unique gene

DIR

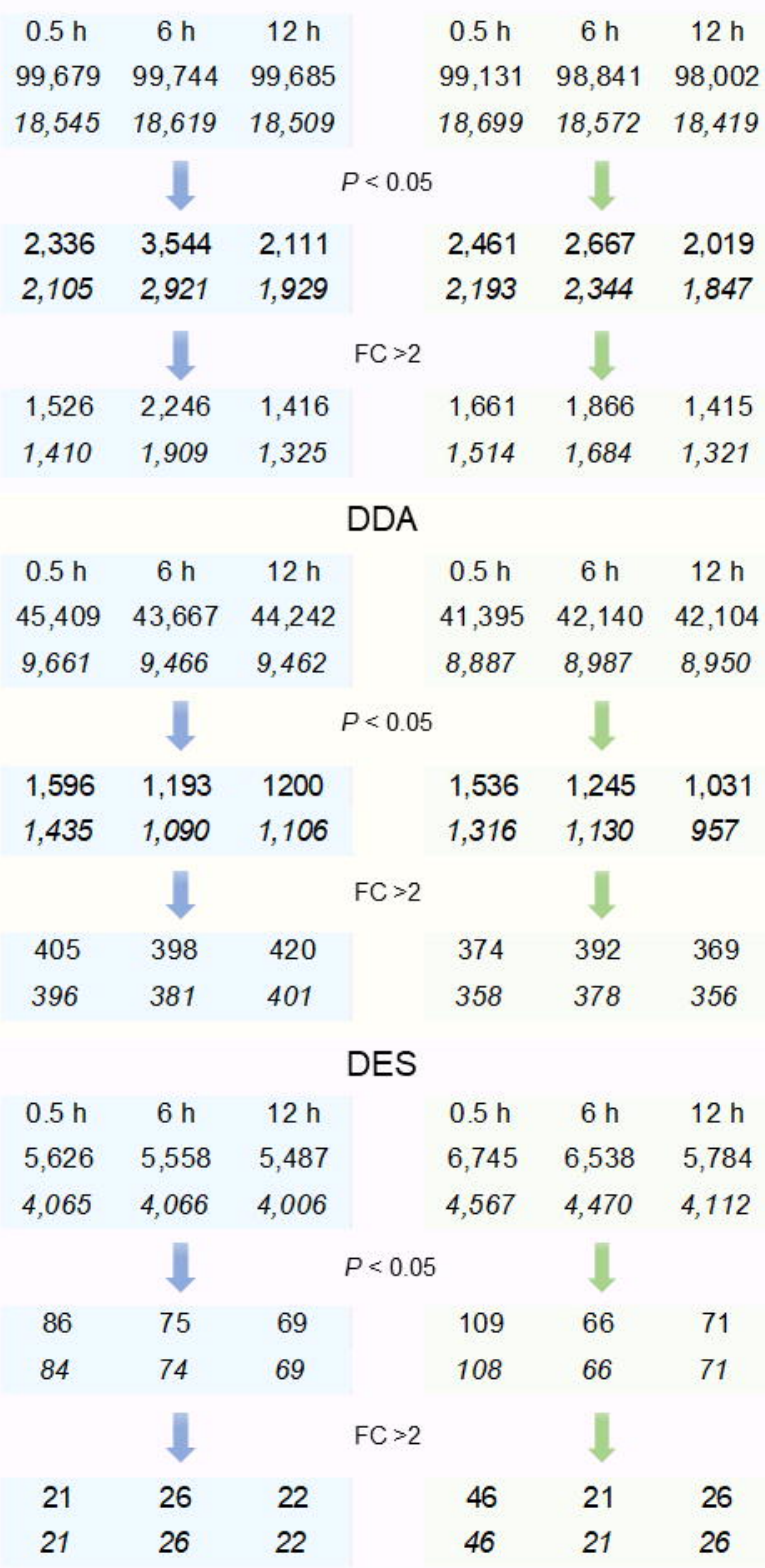

e Roots

Shoots
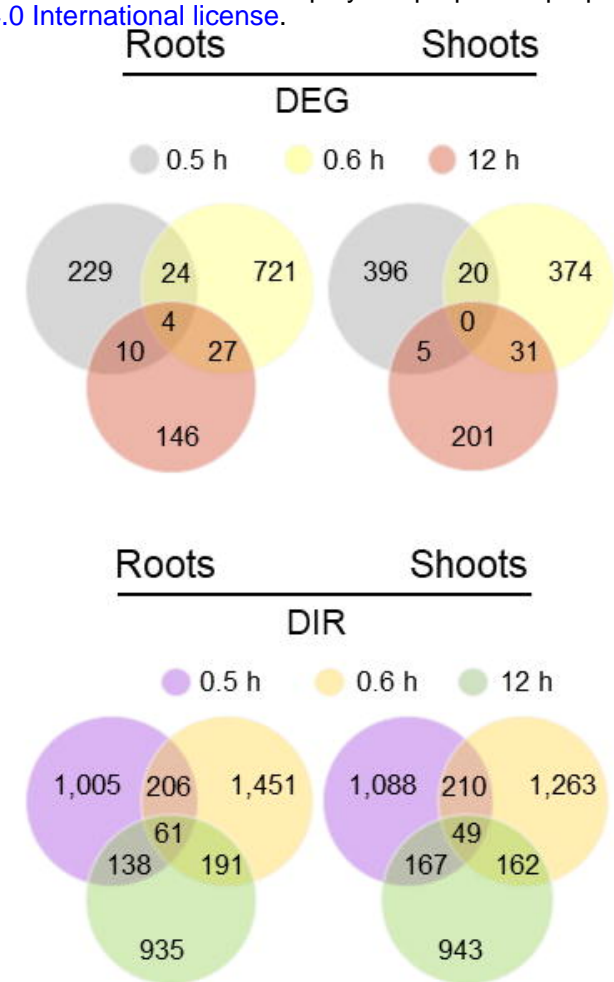

DDA

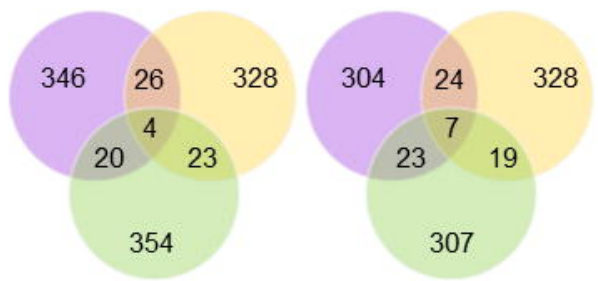

DES
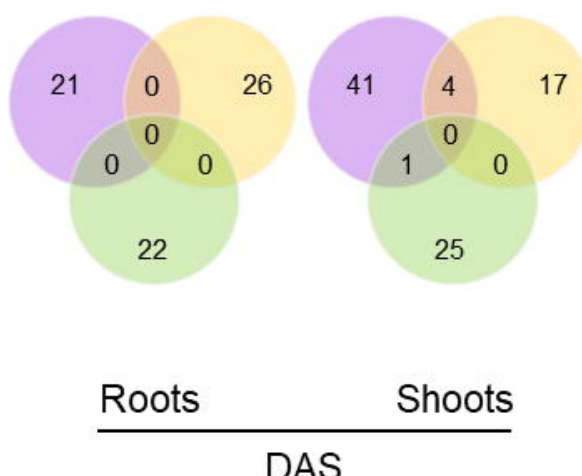

DAS

DIR DDA DES
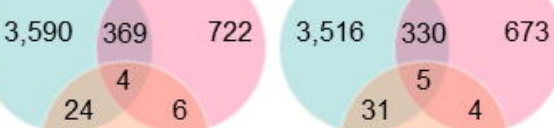

34

48

DAS

DEG

Roots Shoots 
a

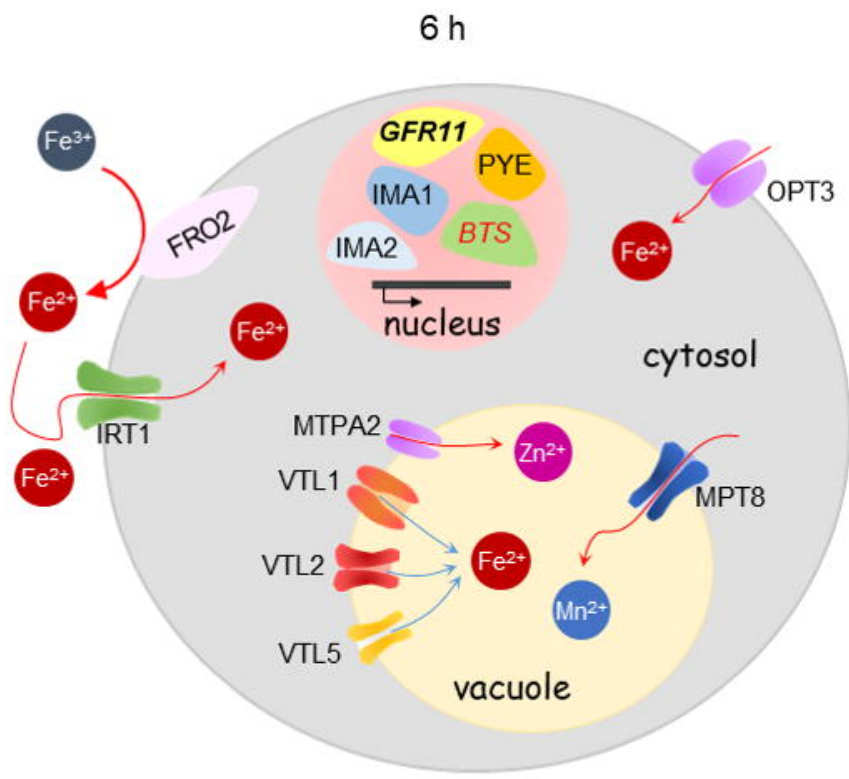

C

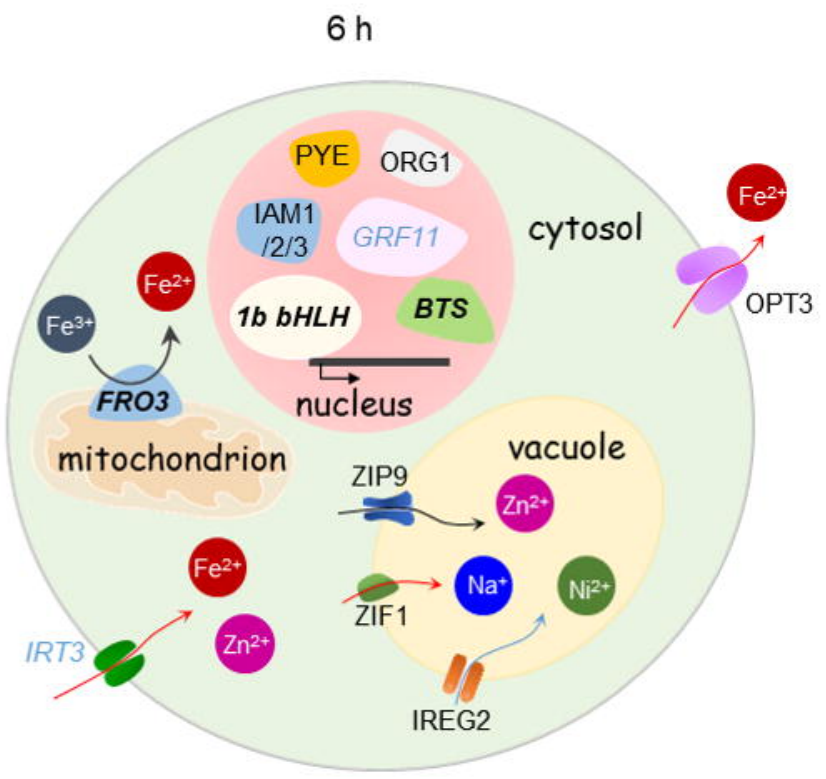

b

$12 \mathrm{~h}$
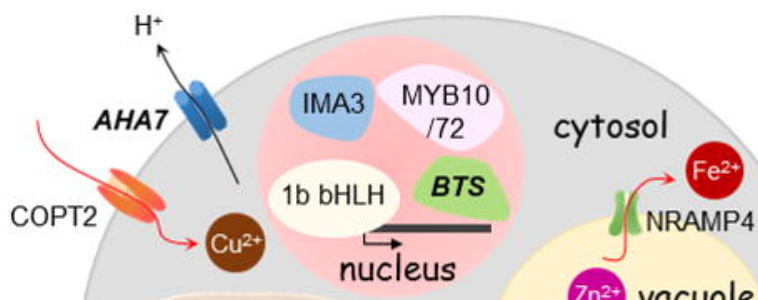
mitochondrion $\quad \mathrm{Zn}^{2+}$ vacuole
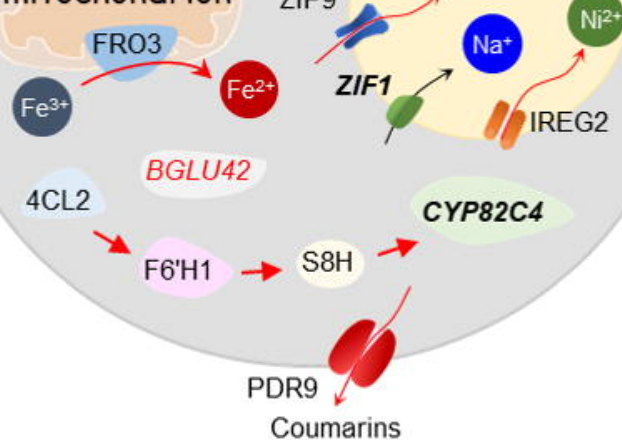

$12 \mathrm{~h}$

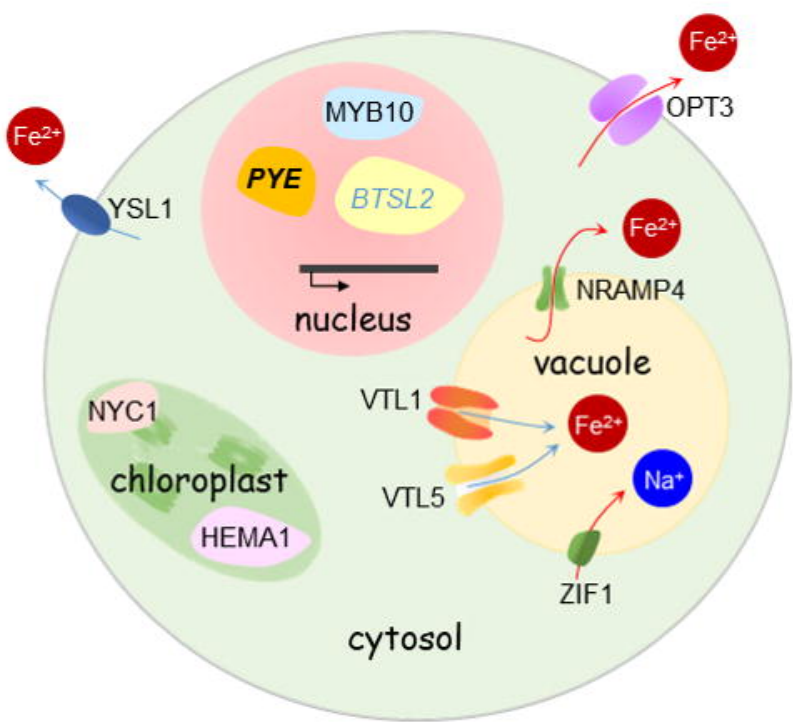




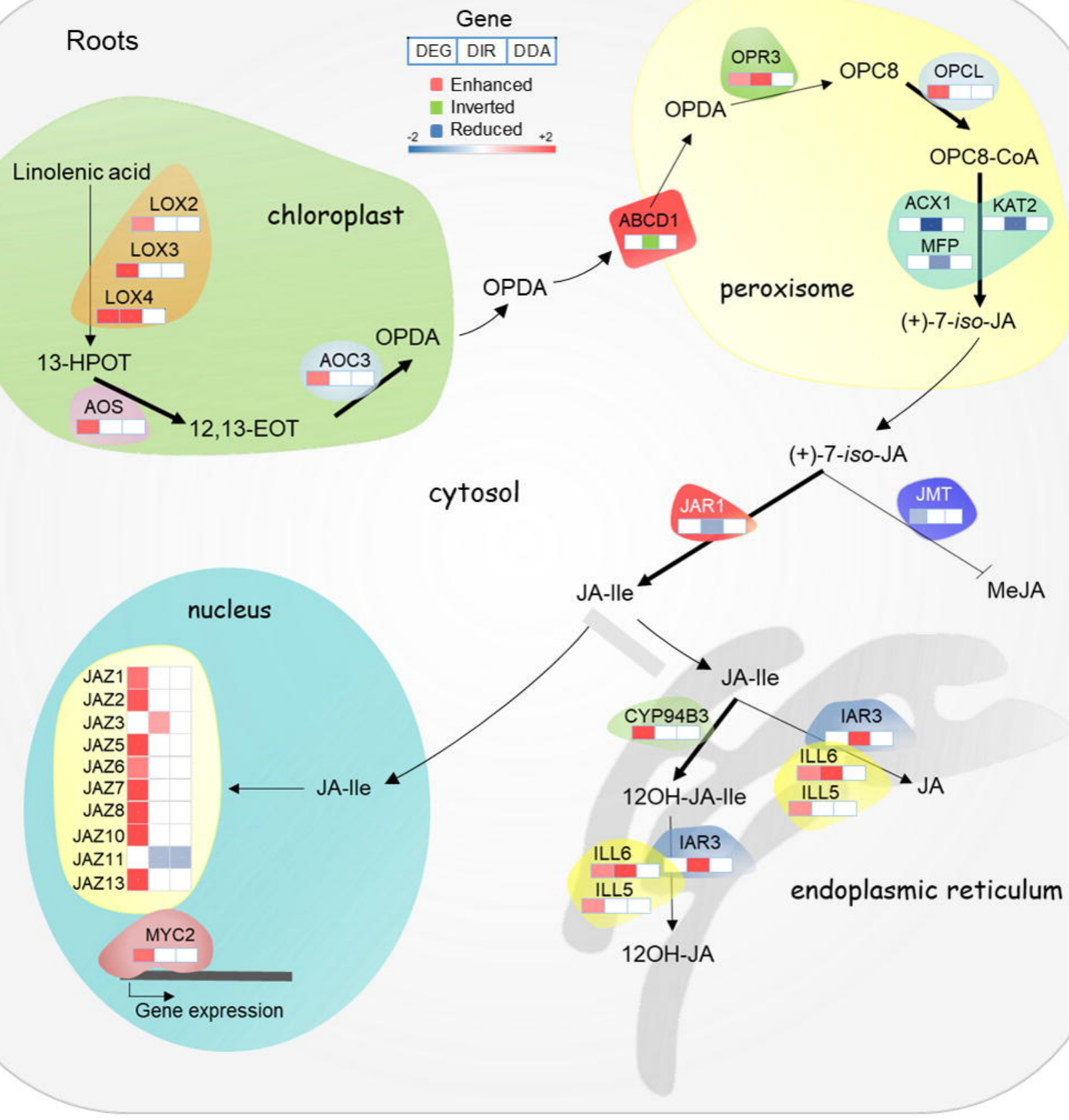


a

\begin{tabular}{|c|c|c|}
\multicolumn{4}{c}{ Gene } \\
\hline DEG & DIR & DDA \\
\hline \multicolumn{2}{|c|}{ Enhanced } \\
\hline & Reduced +2 \\
\hline
\end{tabular}

Roots

Glucose
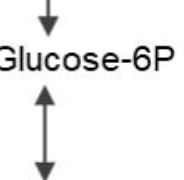

Fructose-6P

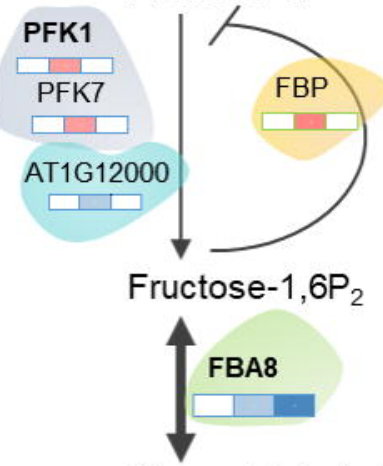

Glyceraldehyde-3P

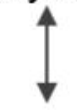

Glycerate- $1,3 \mathrm{P}_{2}$

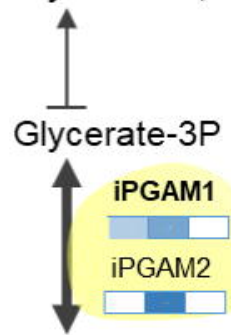

Glycerate-2P

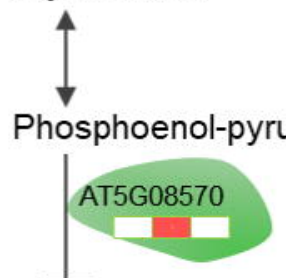

Pyruvate b
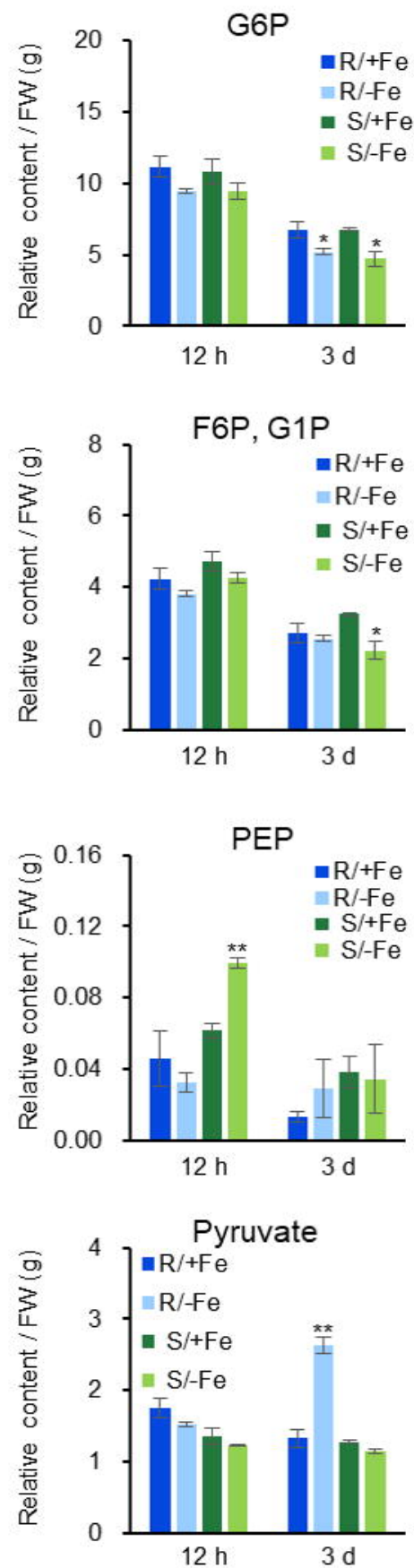

C

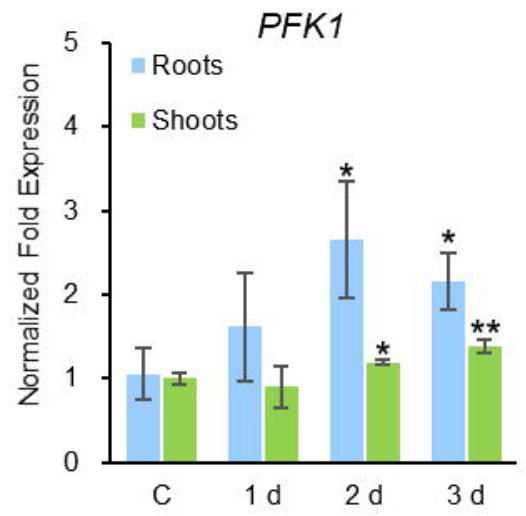

d

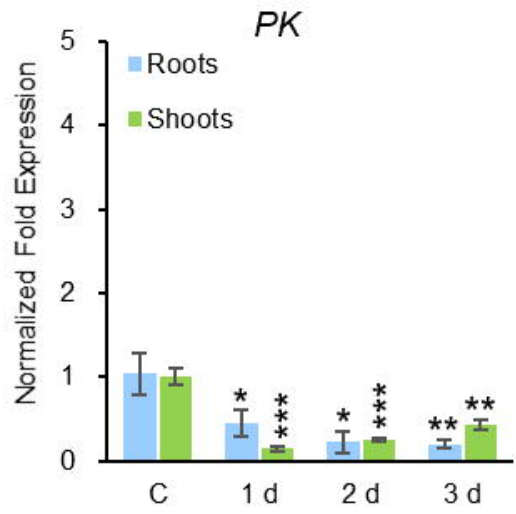

e

\section{bHLH38}

Roots

Shoots

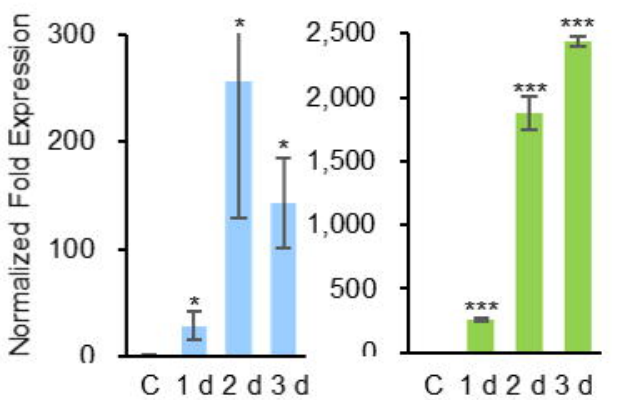


a

Roots

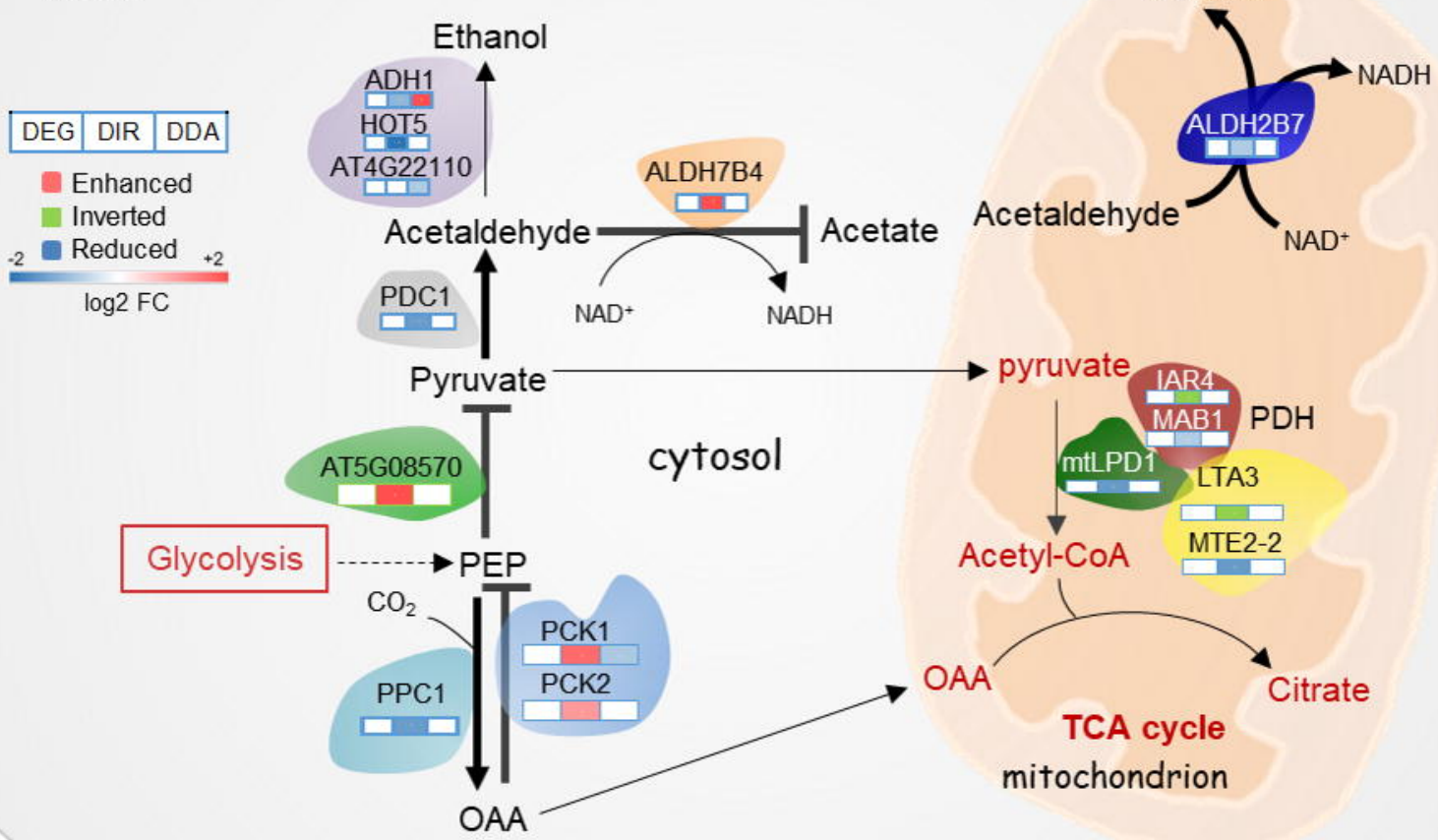

\section{Shoots}

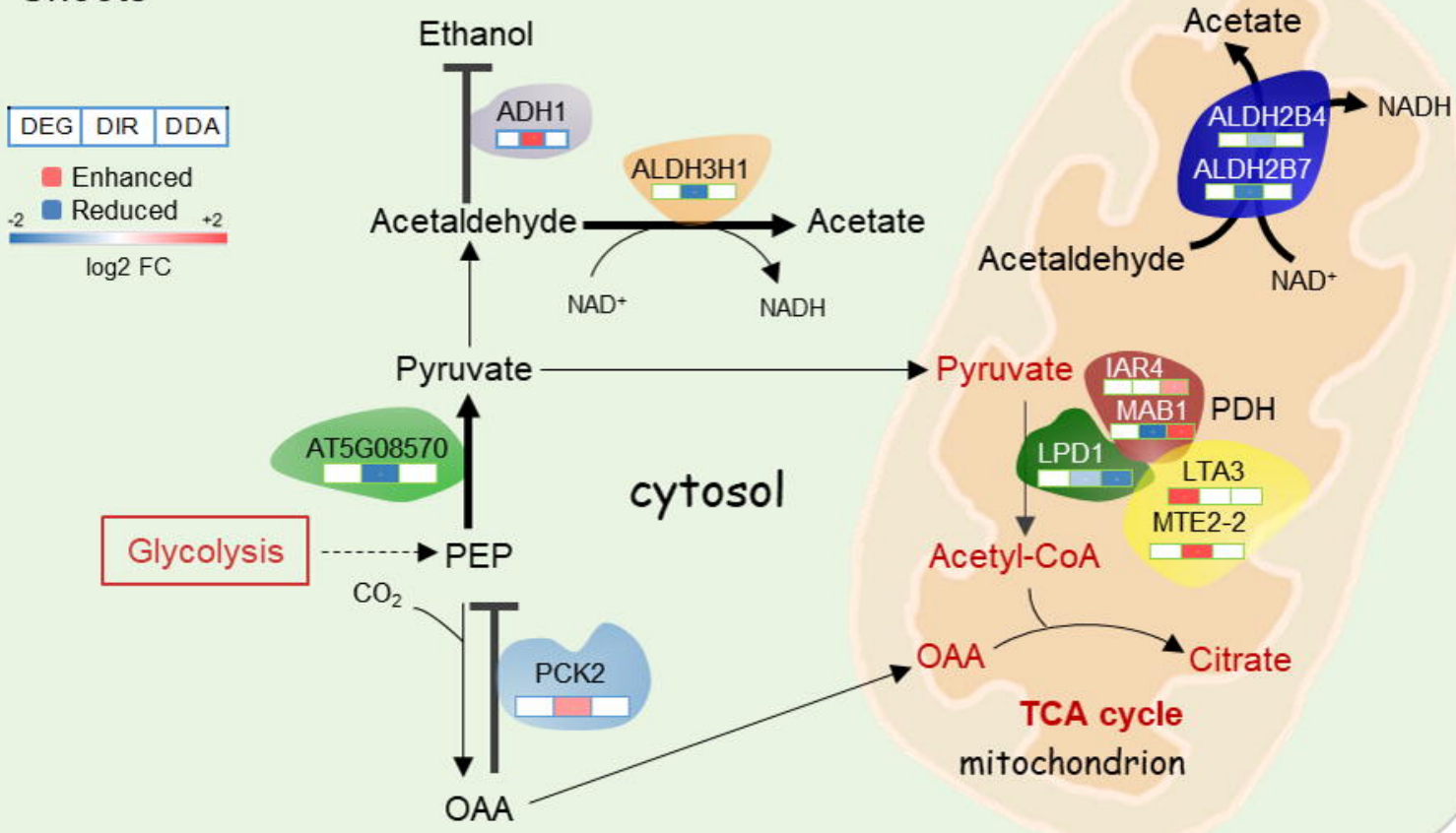


a

DEG vs DIR

Roots

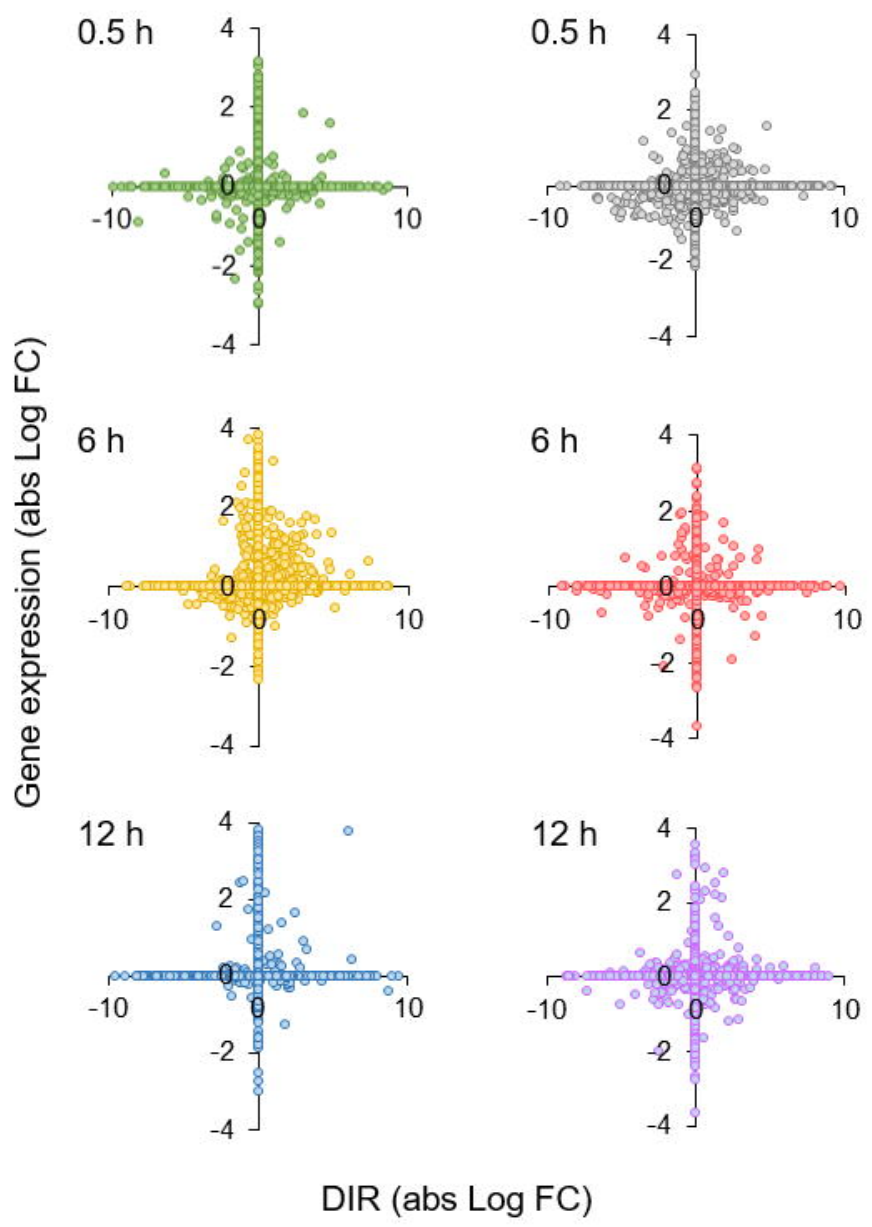

b

Shoots

Roots
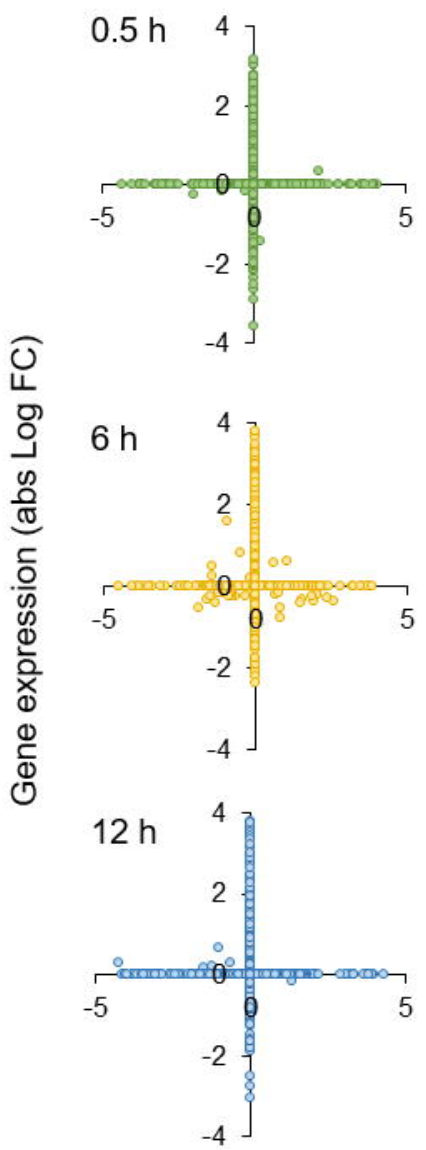

Shoots
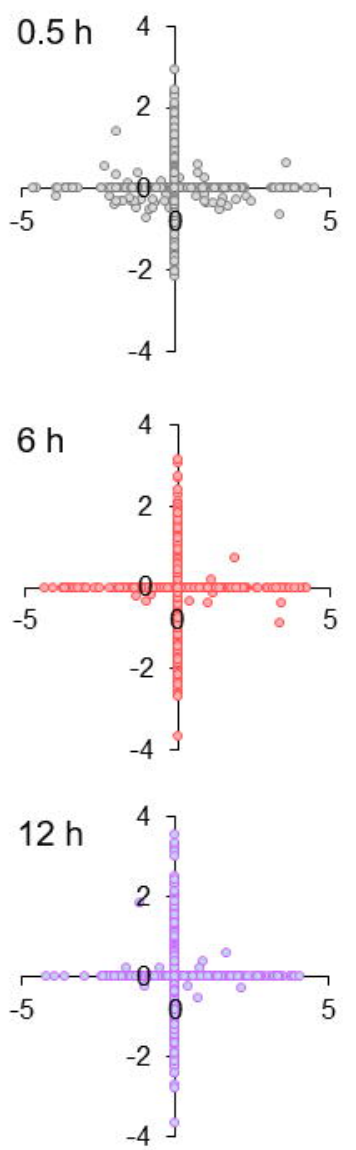

DDA (abs Log FC) 

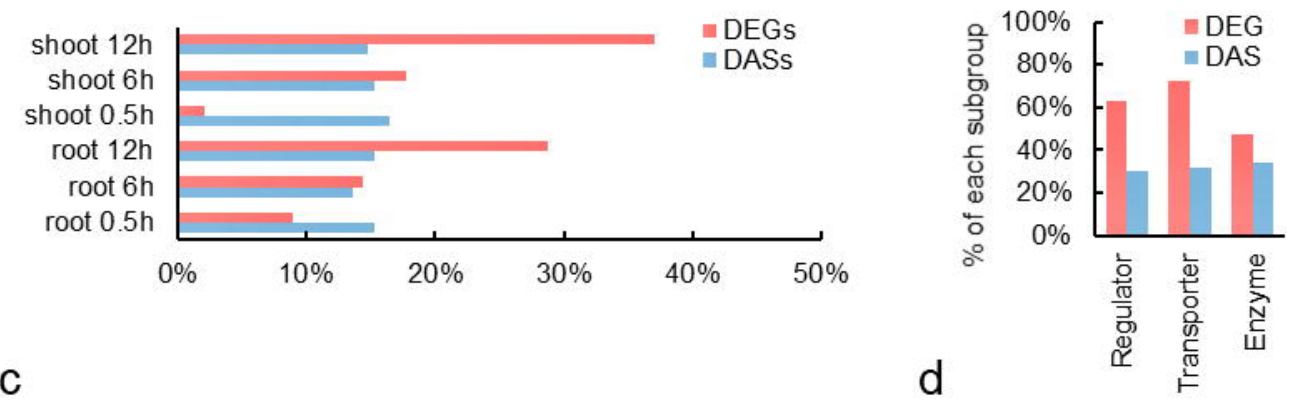

C
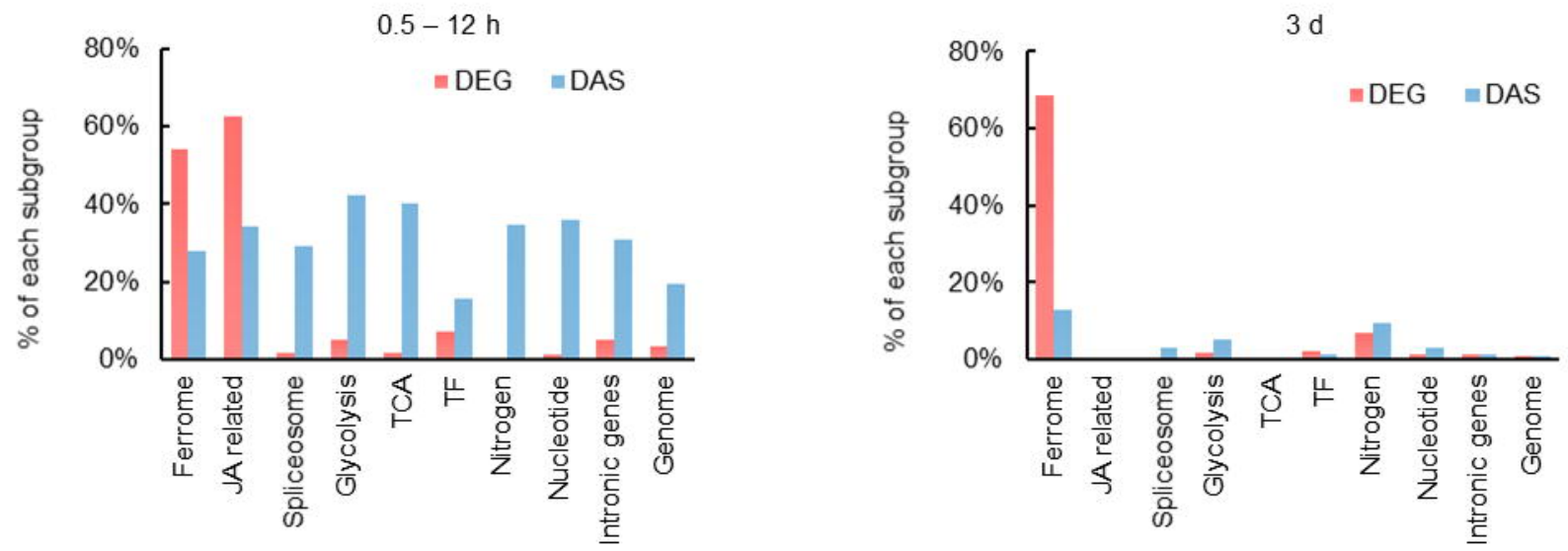
a

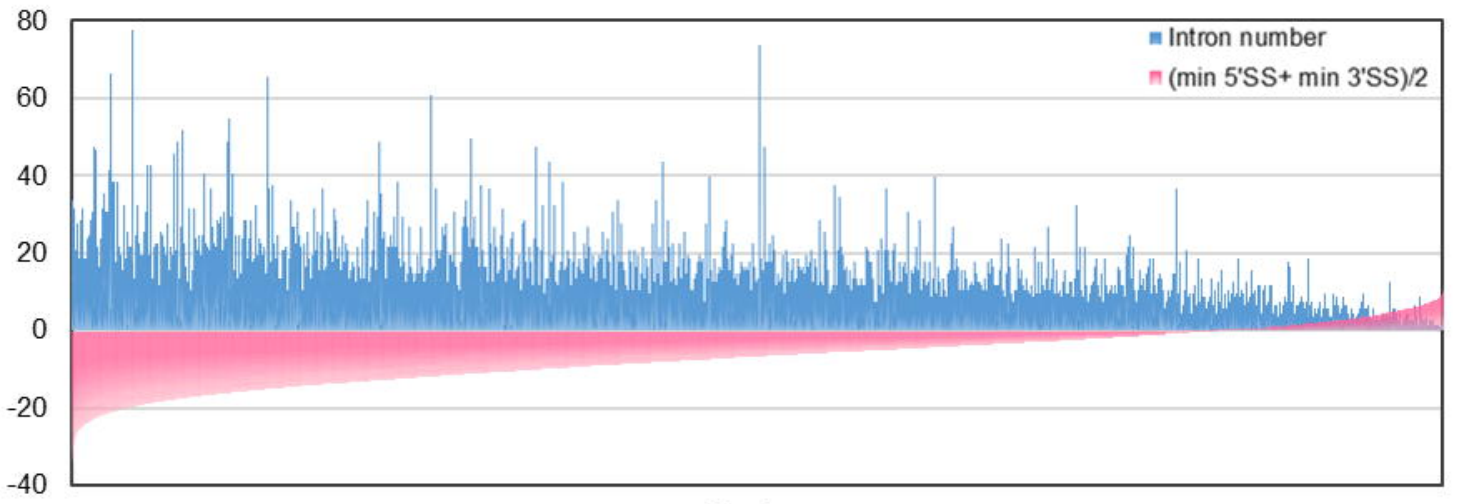

b

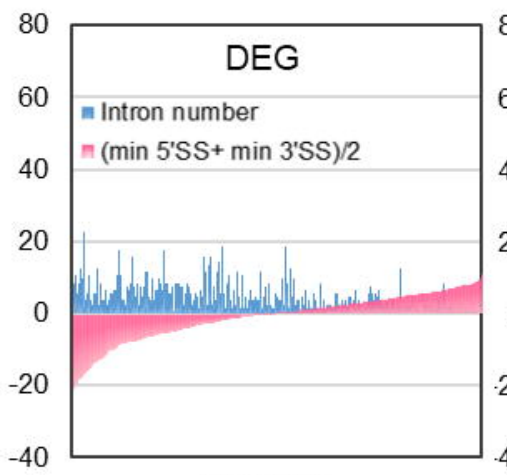

C

Each gene

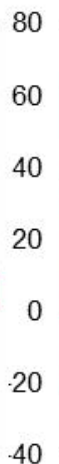

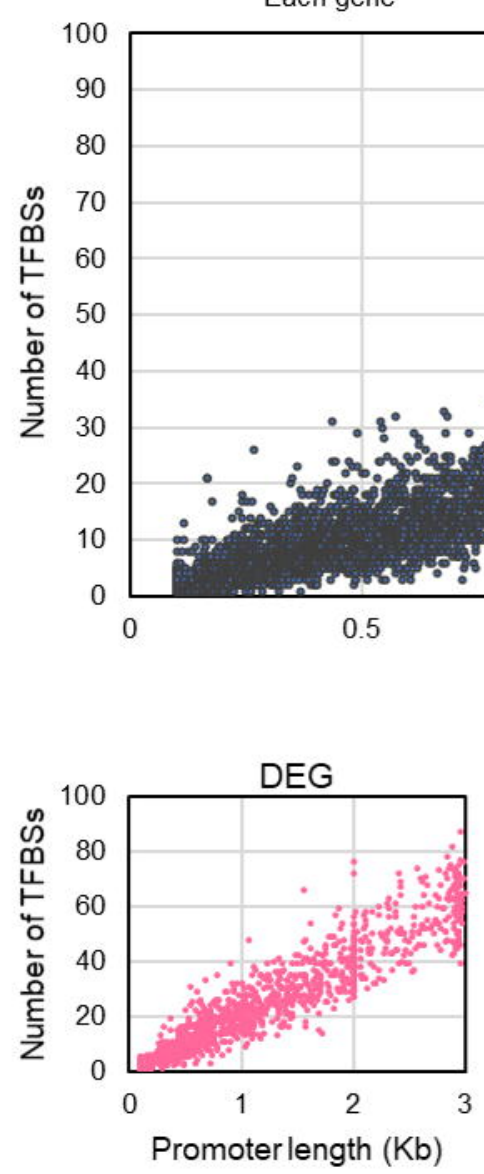
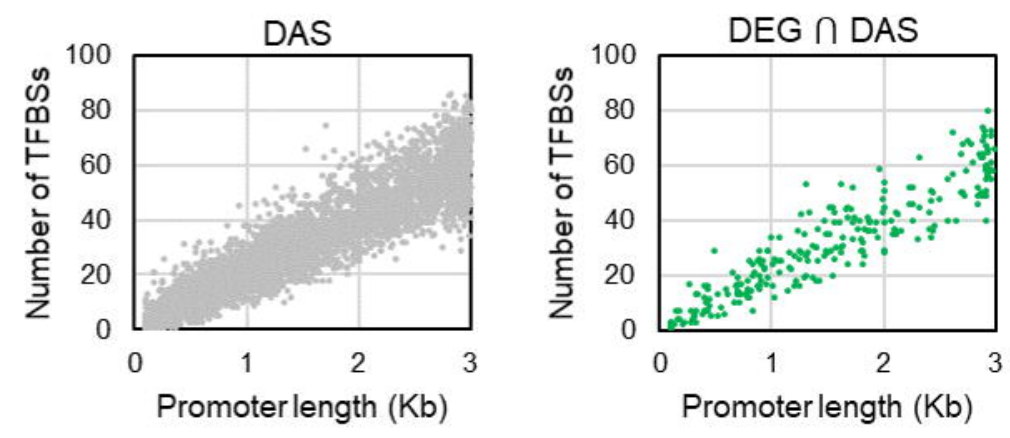

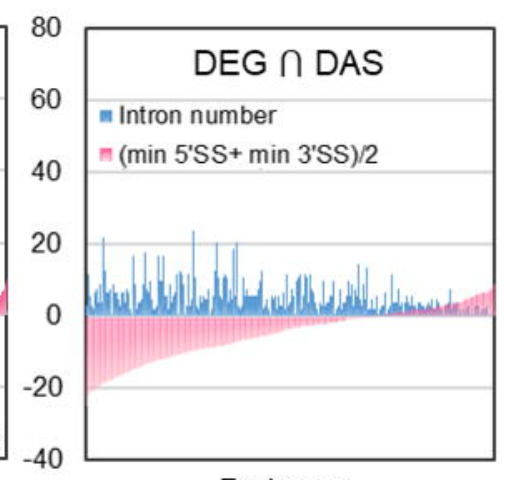

Each gene

Each gene
Each gene 
a

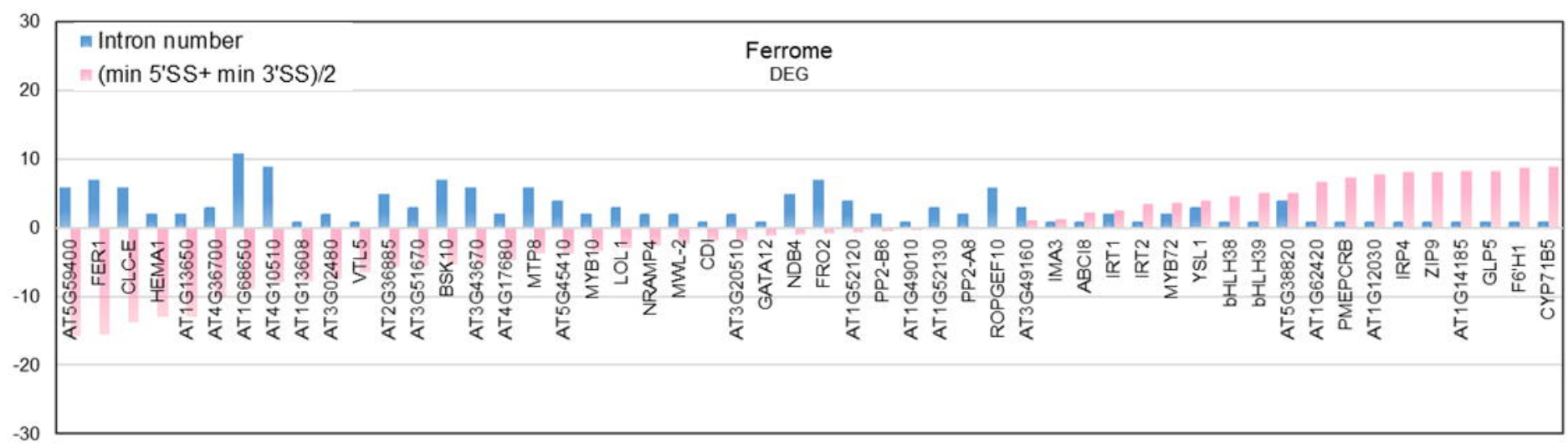

b
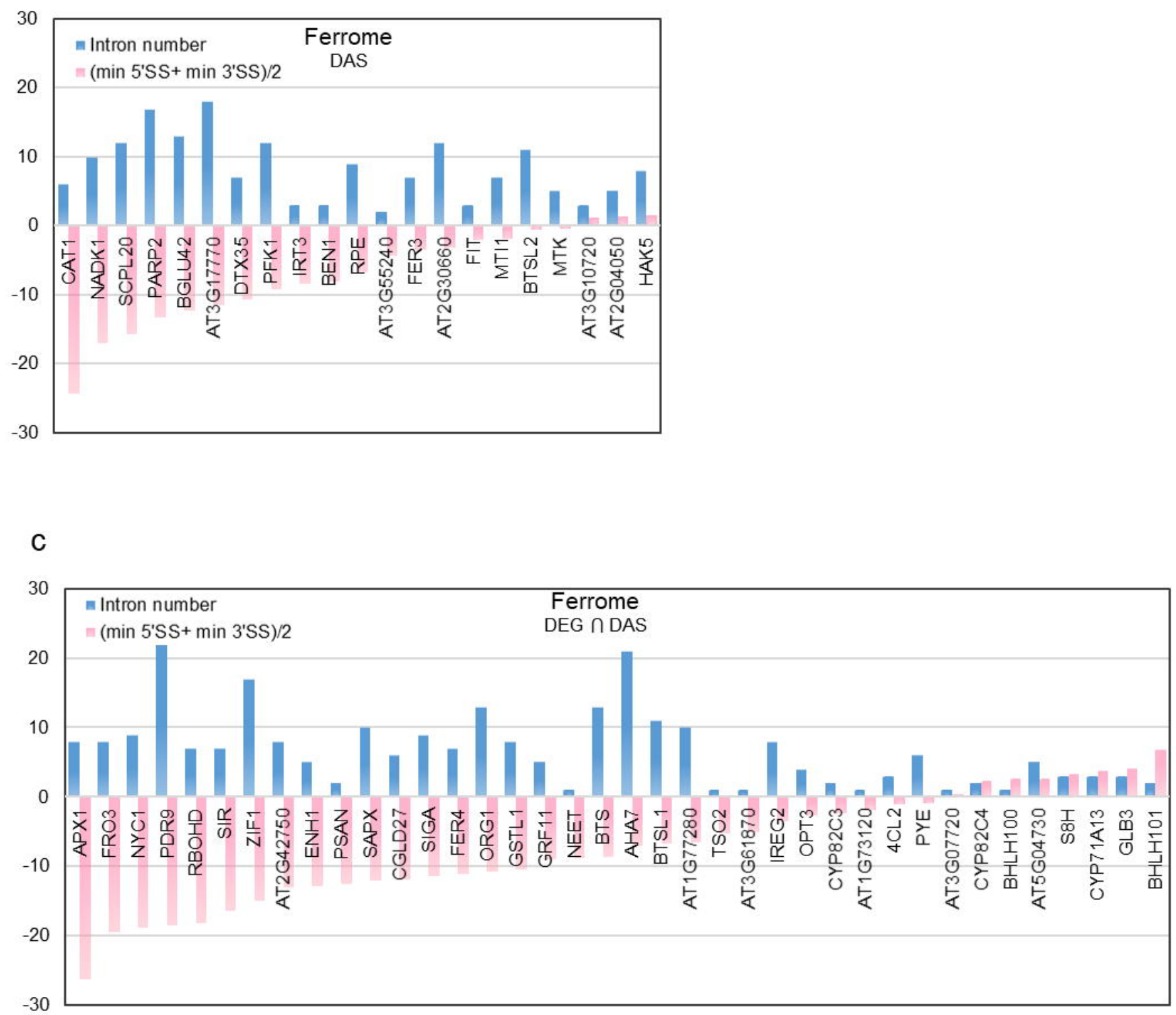
all $\mathbf{E}$ Ferrome $\square$ Glycolysis $\quad$ TCA $\mathbf{I}$ JA $\square$ Spliceosome $\square$ TF

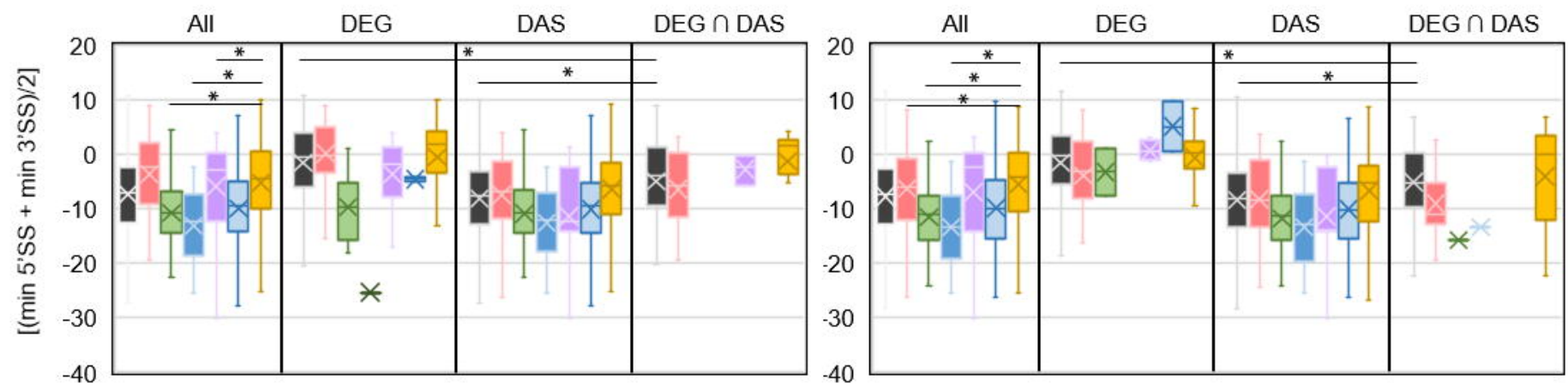

b
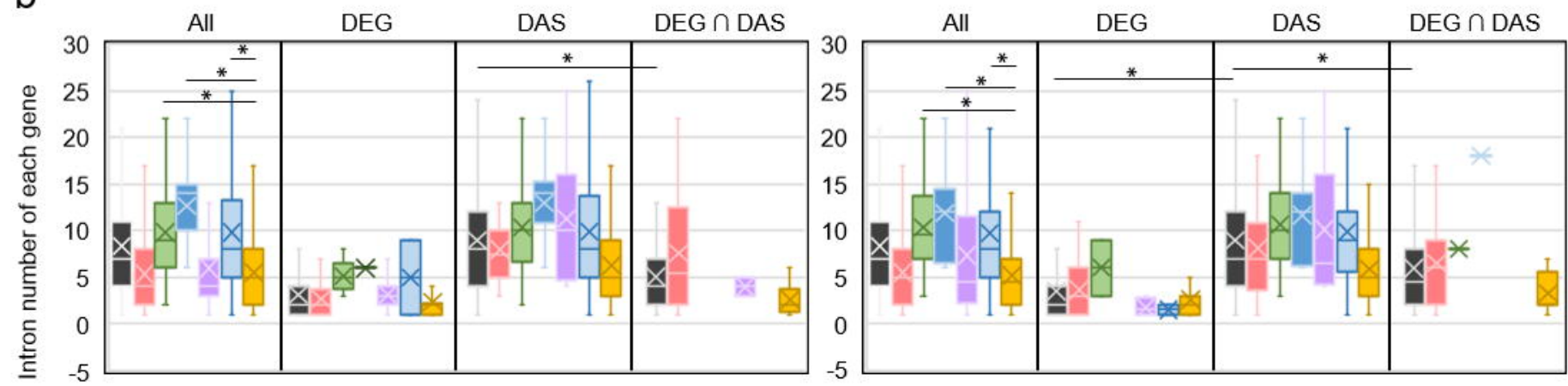

C
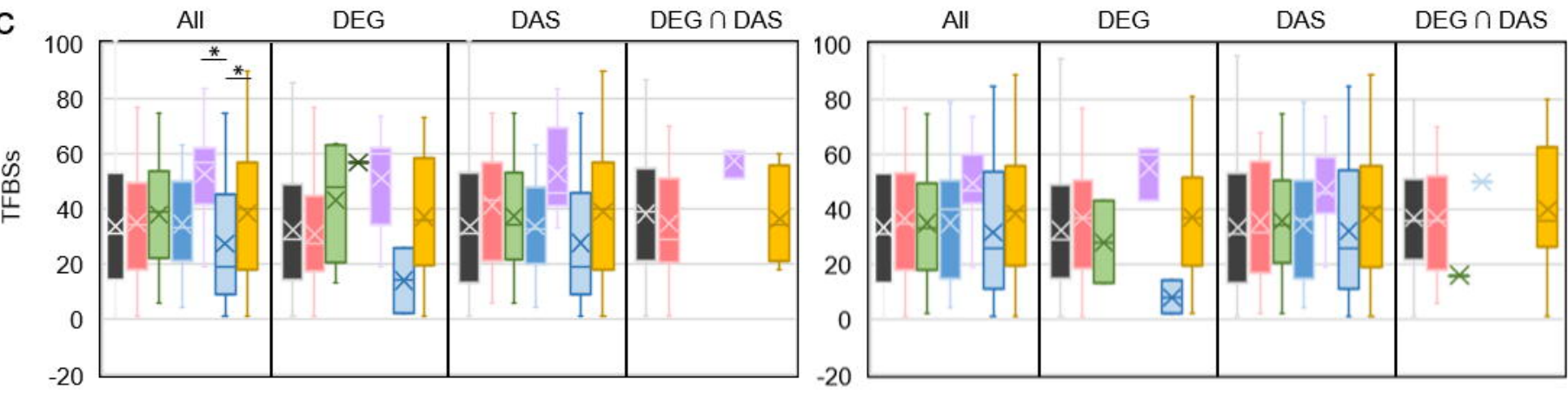

d
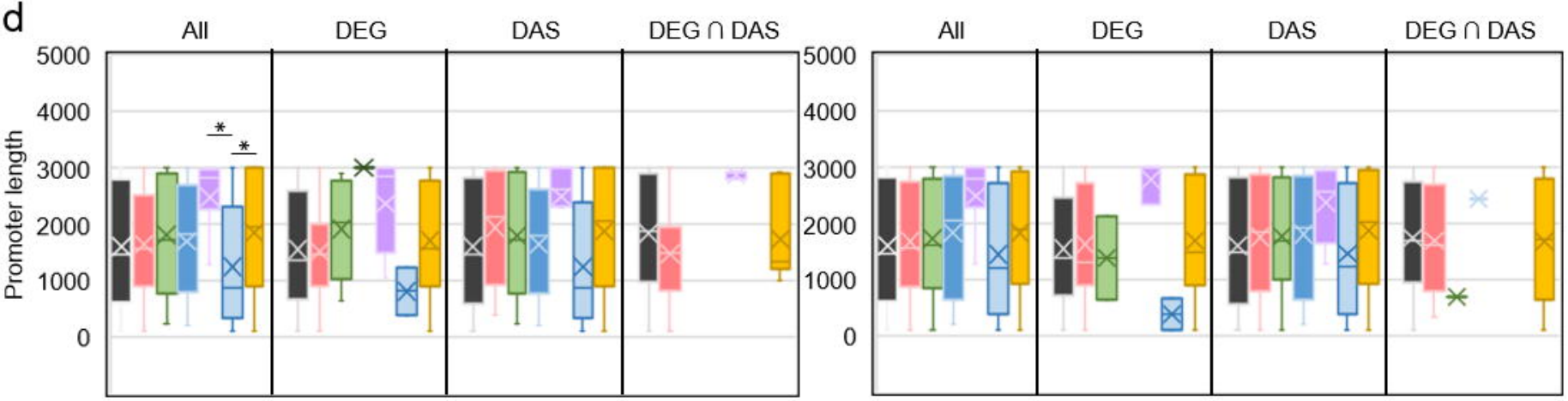


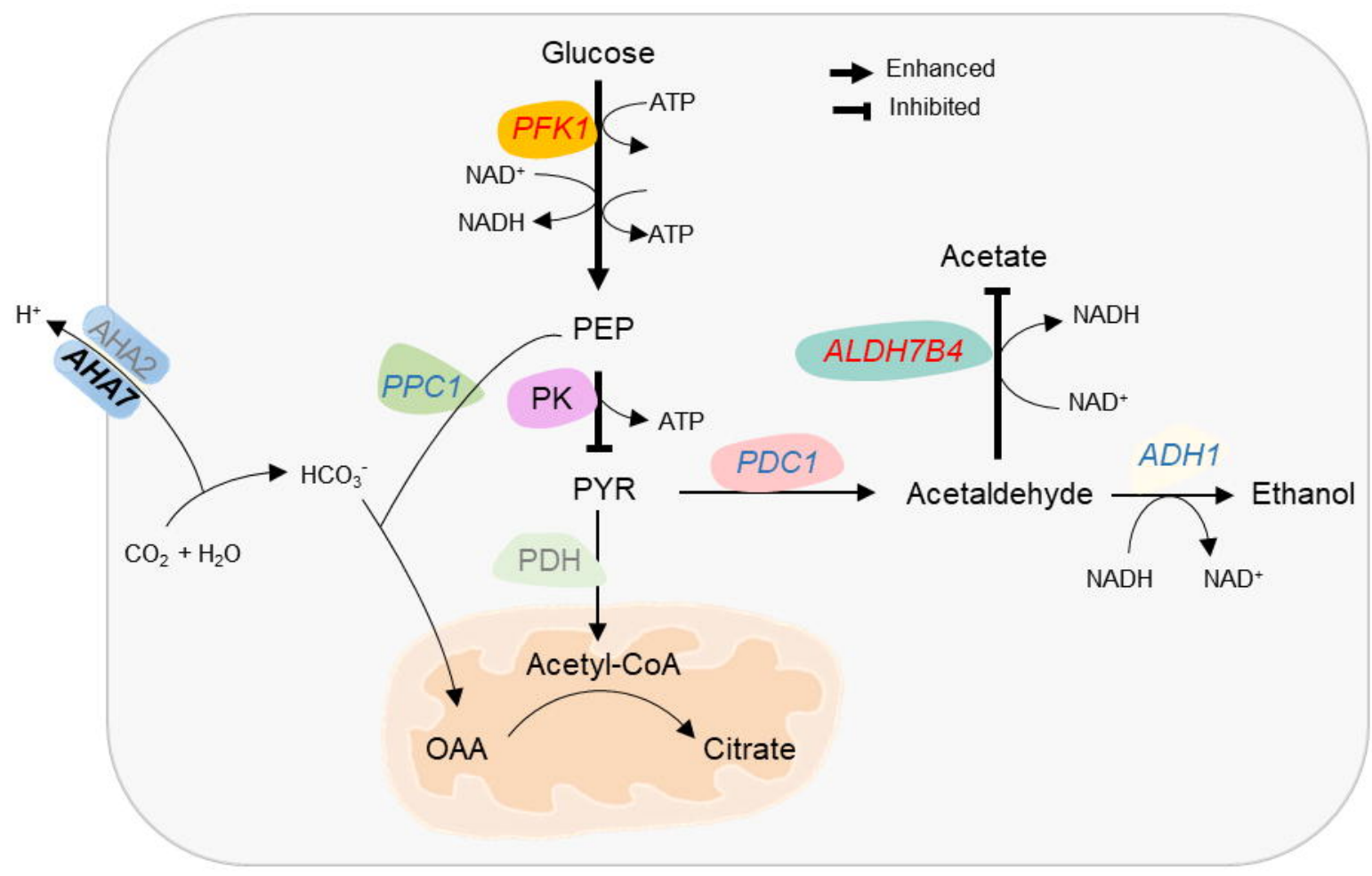

